University of Louisville

ThinkIR: The University of Louisville's Institutional Repository

Electronic Theses and Dissertations

$5-2021$

\title{
CFD analysis of acoustofluidic channels and the effects on biologic delivery.
}

Zachary T. Long

University of Louisville

Follow this and additional works at: https://ir.library.louisville.edu/etd

Part of the Biomedical Engineering and Bioengineering Commons

\section{Recommended Citation}

Long, Zachary T., "CFD analysis of acoustofluidic channels and the effects on biologic delivery." (2021). Electronic Theses and Dissertations. Paper 3445.

https://doi.org/10.18297/etd/3445

This Master's Thesis is brought to you for free and open access by ThinkIR: The University of Louisville's Institutional Repository. It has been accepted for inclusion in Electronic Theses and Dissertations by an authorized administrator of ThinkIR: The University of Louisville's Institutional Repository. This title appears here courtesy of the author, who has retained all other copyrights. For more information, please contact thinkir@louisville.edu. 


\author{
By \\ Zachary T. Long \\ B.S. Bioengineering, University of Louisville, May 2020
}

\author{
A Thesis \\ Submitted to the Faculty of the \\ University of Louisville \\ J.B. Speed School of Engineering \\ As Partial Fulfillment of the Requirements \\ for the Professional Degree
}

\title{
MASTER OF ENGINEERING
}

Department of Bioengineering

April 2021 

Submitted by:

Zachary Long

A Thesis Approved on:

$4 / 6 / 21$

By the following Reading and Examination Committee:

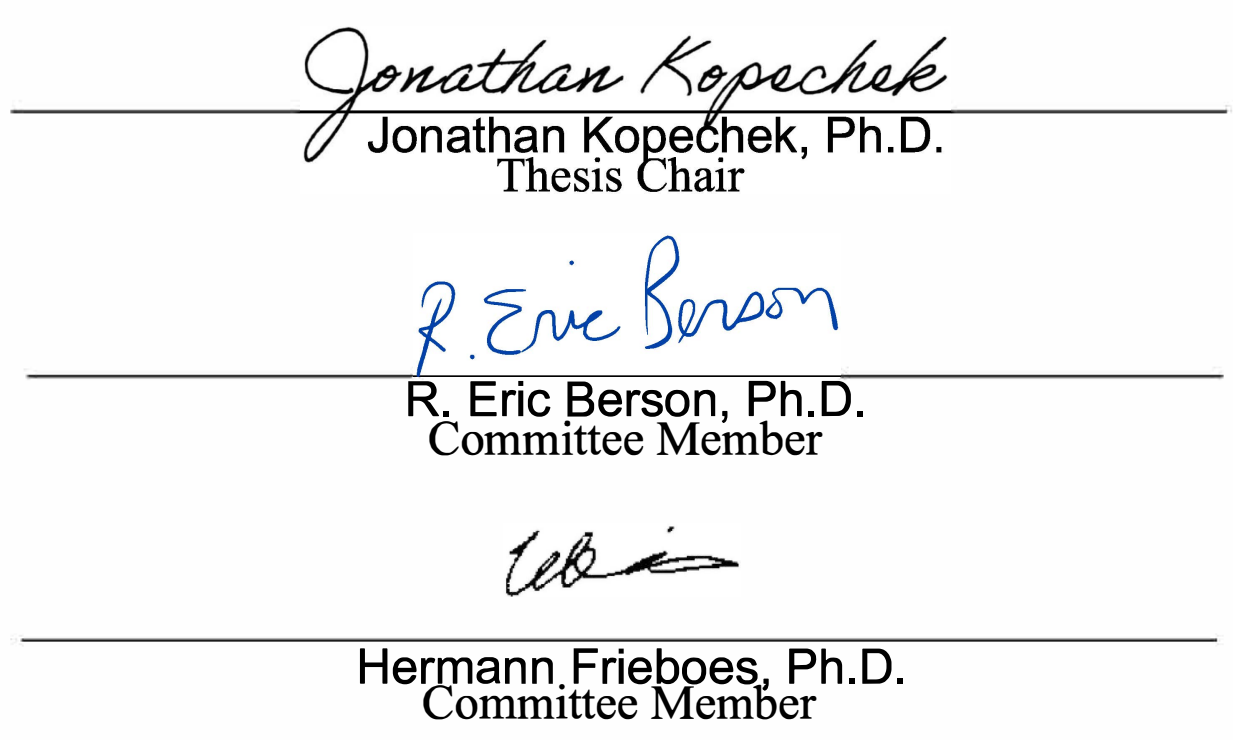

Committee Member

Committee Member 


\section{ACKNOWLEDGEMENTS}

I would first thank to those J.B. Speed School faculty who have agreed to serve on my thesis committee for their support and input on this thesis to ensure it meets all the criterion of adding to the body of scientific knowledge. Having no previous experience with writing for work outside the classroom, the guidance offered by the following committee members was invaluable:
Dr. Jonathan Kopechek
Dr. Hermann Frieboes
Dr. Robert Berson

Additionally, I want to thank all the members of Dr. Jonathan Kopechek's Theranostics Ultrasound Laboratory for all the help in completing the biologic delivery experiments despite my inability to come into the lab with COVID-19 protocols. Their detailed notes, thorough data analysis, and time to teach me made it possible to include "real" results in this thesis, which only adds to the depth achieved. Those lab members include:
Mariah Priddy
Connor Center
Mary Baxter

This thesis would not have been possible without the time and expert knowledge on CFD of Jacob Miller. Jacob, thank you for dealing with my endless stream of questions through email, Microsoft Teams, and text message. Your willingness to meet with me into the late evening hours and weekends is one of the core reasons I was able to complete my thesis. I cannot wait to hear about the success of your Ph.D. defense in a few years - no matter if you go into teaching, industry, or something else, you will be an extraordinary asset.

Finally, I must thank my friends and family for their support and motivation. COVID created a very interesting and difficult year, and there were many times I stumbled, but those closest to me picked me back up and helped me through the journey. 


\begin{abstract}
T-cell transformation is an ever-expanding treatment for several types of cancer, with a potential to be adapted to other disorders in which the immune system plays a key role in the pathophysiology. Currently, all FDA approved chimeric antigen receptor (CAR) T-cell cancer therapies rely on transformation via viral transduction. However, viral transduction is plagued by poor consistency and the potential to create adverse immune reactions when T-cells are reintroduced into a patient. Other transformation methods are being explored, with an alternative called acoustofluidic sonoporation showing promise. In these procedures, cells are passed through a channel, of the millimeter scale, while ultrasound (US) is applied. The US causes unstable cavitation of perfluorocarbon microbubbles (MBs) resulting in rupture that reversibly permeabilizes cells, allowing entry of almost any water-soluble biologic (e.g. DNA/RNA, small molecules, etc.). While current research demonstrates that acoustofluidic sonoporation may be better than other transfection methods, there is a limited understanding of the fluid dynamics within the acoustofluidic devices and the physical mechanisms of the alteration in cell permeability. In this thesis, computational fluid dynamic (CFD) modeling was utilized to simulate fluid and particle flow through various acoustofluidic channel geometries and the results were compared with biological delivery experiments to cells. It was found a 1-mm diameter Concentric Spiral channel is an optimal design as it maximizes wall shear stress (WSS) and US exposure, as compared to 1$\mathrm{mm}$ and 2-mm diameter Rectilinear channels. With further refinement of the CFD simulations, optimization of channel geometry, flow rate, and US parameters could be enhanced. This optimization could enable acoustofluidic sonoporation to be translated into manufacturing of CAR T-cell therapies for clinical treatments of cancer and other disorders in the future.
\end{abstract}




\section{TABLE OF CONTENTS}

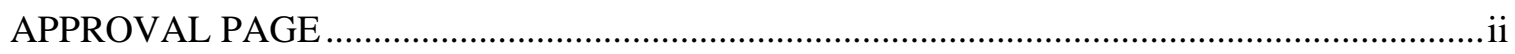

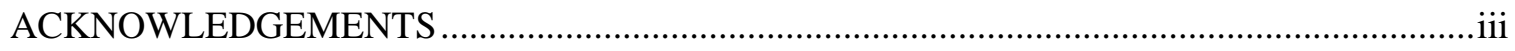

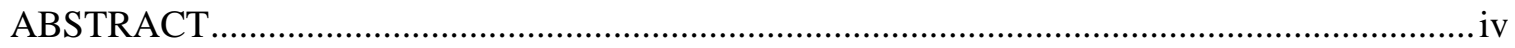

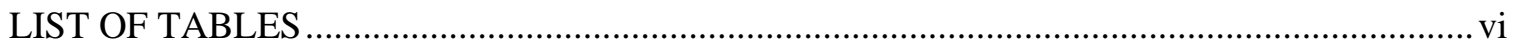

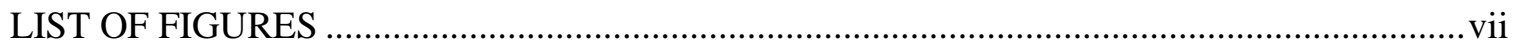

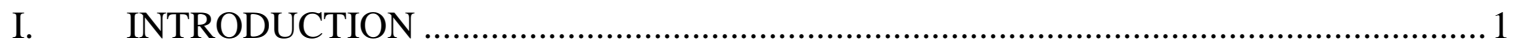

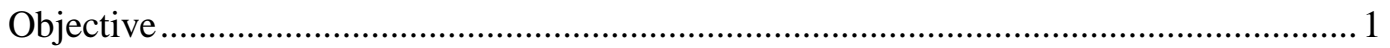

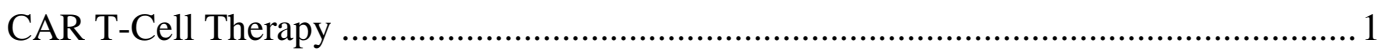

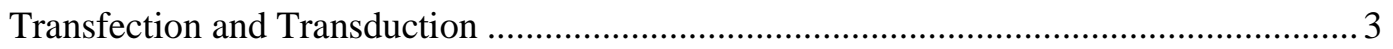

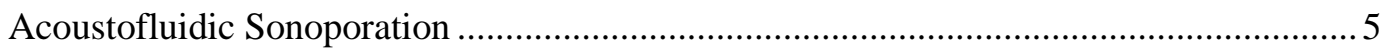

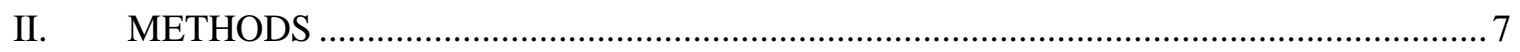

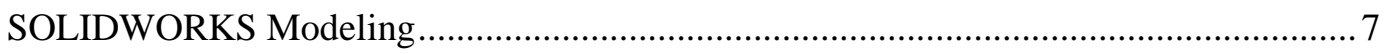

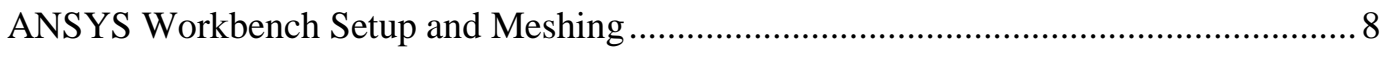

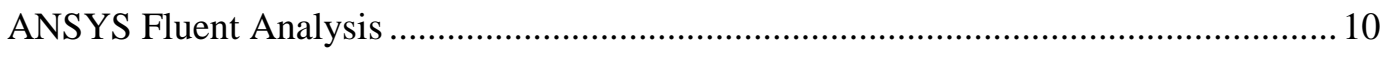

Jurkat T-Cell Culturing, Transfection, and Analysis ................................................... 15

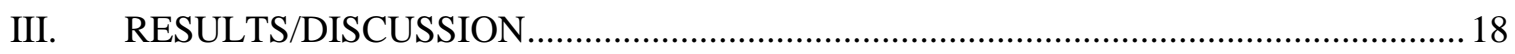

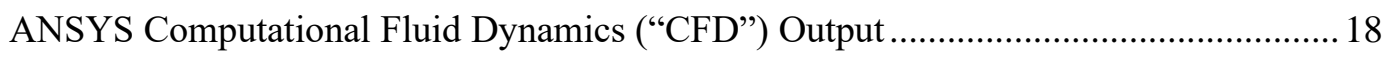

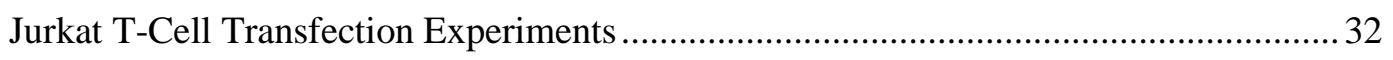

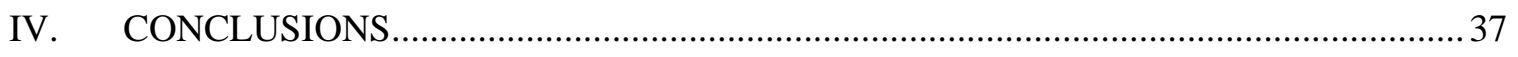

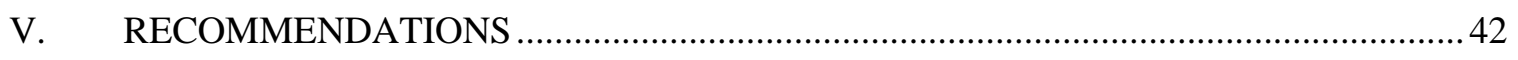

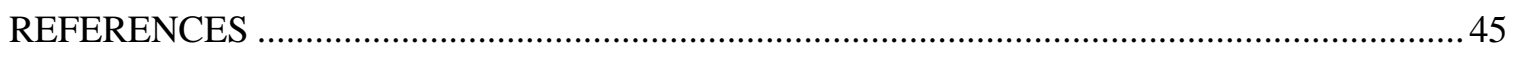

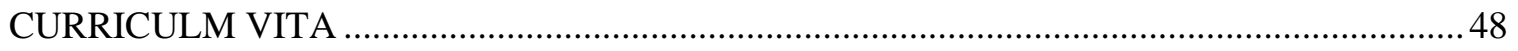




\section{LIST OF TABLES}

$\underline{\text { Page }}$

Table 1. Description of transformation methods. .................................................... 3

Table 2. Drawbacks of transformation techniques. .................................................. 4

Table 3. Reynolds number calculations at inlet velocity $=0.002 \mathrm{~m} / \mathrm{s}$. ...................... 11

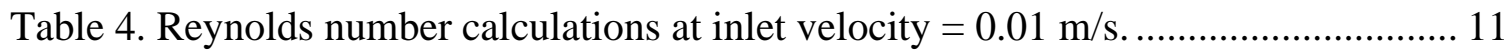

Table 5. Selected characteristics for each channel at the optimal mesh size.................. 15

Table 6. Volume average WSS values at each inlet velocity ..................................... 19

Table 7. Volume average WSS values at each inlet pressure.................................... 20

Table 8 . Volume average velocity values at each inlet velocity ................................. 24

Table 9. Volume average velocity values at each inlet pressure ................................. 25

Table 10. Minimum and maximum microbubble velocity values............................... 27

Table 11. Microbubble residence time histogram metrics .......................................... 30

Table 12. Biologic delivery results for the first cell experiment ................................ 32

Table 13. Cell viability results for the first cell experiment .................................... 32

Table 14. Biologic delivery results for the second cell experiment .............................. 34

Table 15. Cell viability results for the second cell experiment................................... 34 


\section{LIST OF FIGURES}

$\underline{\text { Page }}$

Figure 1. Graphical depiction of the CAR T-cell process ......................................... 2

Figure 2. Renderings of the channels in SOLIDWORKS ...................................... 7

Figure 3. Labeled named selections (inlet,outlet, fluid flow) .................................... 8

Figure 4. Schematic regions labeled where the Reynolds number was calculated........... 10

Figure 5. Mesh independence analyses for all the channel geometries ......................... 14

Figure 6. Image of acoustofluidic sonoporation setup for cell experiments .................... 16

Figure 7. Scaled WSS contours for each channel geometry ….................................. 18

Figure 8. Comparison of WSS for each channel geometry at two inlet velocities .......... 19

Figure 9. Comparison of WSS for each channel at both inlet pressures........................ 20

Figure 10. Scatterplot of inlet velocity effect on WSS for each channel....................... 21

Figure 11. DPM Concentration contours for each of the channel geometries ................. 22

Figure 12. Scaled velocity pathline contours for each channel geometry ...................... 23

Figure 13. Comparison of average velocity for each channel at two inlet velocities ....... 24

Figure 14. Comparison of average velocity for each channel at both inlet pressures ...... 25

Figure 15. Scaled microbubble (MB) velocity contours for each channel geometry ....... 26

Figure 16. Comparison of $\min / \max$ microbubble velocity for each channel geometry ... 27

Figure 17. Images of the particle distribution at the outlet of each channel ................... 29

Figure 18. Scaled contours of microbubble residence time for each channel geometry .. 29

Figure 19. Histograms of particle residence time for each channel.............................. 30

Figure 20. Unscaled images of microbubble velocity, focused on outlet variation.......... 31 
Figure 21. Calcein fluorescence and percent viability for the first cell experiment ........ 32

Figure 22. Calcein fluoroscence and percent viability for the second cell experiment .... 33

Figure 23. Association of WSS and calcein fluorescence ........................................ 35

Figure 24. Potential design for channel with multiple $180^{\circ}$ turns ................................ 37 


\section{INTRODUCTION}

\section{Objective}

The objective of this thesis is to analyze the fluid flow in acoustofluidic channels to understand the fluidic forces acting on cells during sonoporation procedures.

\section{CAR T-Cell Therapy}

Cancer is caused by unregulated cell growth and inability of the immune system to remove these cells. Standard cancer treatments include chemotherapy, radiation therapy, and surgery. However, each of these treatments can take a significant physical and mental toll on the patient, usually require repeated/regular treatment, and are rarely $100 \%$ effective. Current cancer treatments can drastically affect a patient's quality of life and therefore a new treatment modality is needed.

Chimeric antigen receptor (CAR) T-cell therapy is a newer, and very promising, cancer treatment. There are several FDA approved CAR T-cell treatments currently, with several others being explored. Three current, CAR T-cell therapies are discussed briefly below:

1) Tecartus (brexucabtagene autoleucel) - utilizes a gammaretrovirus vector to modify Tcells to bind and kill relapsed or refractory mantle cell lymphoma ${ }^{[3]}$

2) Kymriah (tisagenlecleucel) - utilizes a lentiviral vector to modify T-cells to recognize relapsed or refractory diffuse large B-cell lymphoma and relapsed or refractory acute lymphoblastic leukemia ${ }^{[1]}$

3) Yescarta (axicabtagene ciloleucel) - utilizes a retroviral vector to alter T-cells to target diffuse large B-cell lymphoma (DLBCL), primary mediastinal B-cell lymphoma, and high-grade B-cell lymphoma selectively ${ }^{[2]}$

All of these therapies have shown a marked effectiveness in patients. During clinical trials, Yescarta had $82 \%$ of patients respond to the treatment, with $54 \%$ reaching complete remission. Follow-up data determined that $40 \%$ of those reaching complete cancer remission remained in complete remission (median follow-up 15.4 months) ${ }^{[17]}$. Kymriah had 53\% of trial participants 
respond to treatment, with $40 \%$ reaching complete remission; no follow-up data was provided ${ }^{[21]}$. Finally, Tecartus saw very similar results as Yescarta: $87 \%$ of patients responded to the treatment with $62 \%$ reaching complete remission ${ }^{[28]}$. From this data, it appears that CAR T-cell therapy, or more generally, tuning of the adaptive immune response, can be an extremely effective treatment.

CAR T-cell therapy is effective because it harnesses the patient's own immune system, making use of their T-cells. To begin, a patient's T-cells are harvested and then genetically altered/modified to express a specific antigen receptor. After reprogramming, the T-cells are expanded in-vitro, reinfused into the patient, and then can specifically and selectively target and kill any cell expressing that antigen through the cell-mediated branch of the adaptive immune system. CAR T-cell therapy offers a specificity and sensitivity that chemo, radiation, or surgery cannot match. Additionally, the body learns to synthesize T-cells with the specific antigen receptor, which builds an active, permanent "immunity" that can reduce or remove the need for repeated treatment. The process of CAR T-cell therapy is detailed in Figure 1.

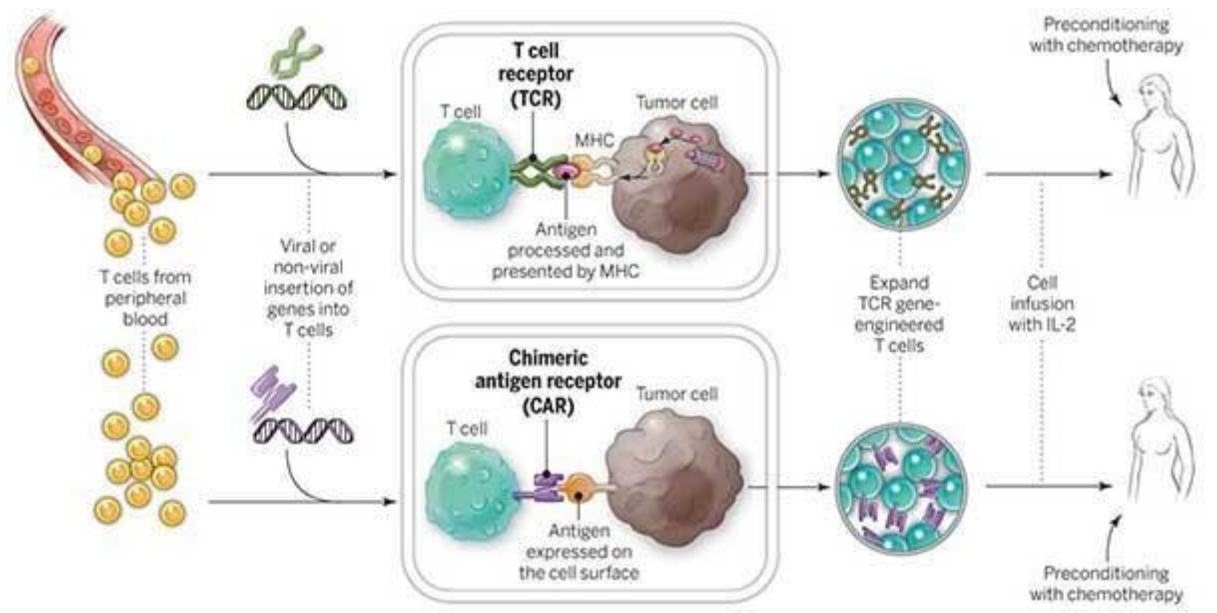

Figure 1. Graphical depiction of the CAR T-cell process from harvesting, transformation, expansion, and reinfusion ${ }^{[7]}$. Once reinfused, the T-cells recognize specific antigens on tumor cells to either directly kill the cell or activate other immune cells to clear the tumor.

It is critical to note that all current FDA approved CAR T-cell treatments rely on viral transduction for T-cell transformation. Viral transduction is only one of many methods available 
for transformation and while the advantages are apparent (no other approved treatment utilizes a different method) there are significant disadvantages associated with viral transduction. More on various methods of transformation are discussed below.

\section{Transfection and Transduction}

Transfection and transduction are processes in which exogenous DNA or RNA are introduced into a cell to alter gene expression and produce recombinant proteins. Transduction specifically relies upon viral vectors to infect cells which can or cannot integrate with the host genome while transfection relies upon non-viral methods such as electroporation, microinjection, cell squeezing, and sonoporation. Descriptions of each technique are provided in Table 1.

Table 1. Description of various transformation methods.

\begin{tabular}{|c|l|}
\hline $\begin{array}{c}\text { Transformation } \\
\text { Technique }\end{array}$ & \multicolumn{1}{c|}{ Description } \\
\hline Viral Transduction & $\begin{array}{l}\text { A specific DNA or RNA sequence is packaged into a viral particle } \\
\text { (virion) that "infects" cells, through endocytosis of the nucleic acid, } \\
\text { which is then transported to the nucleus and expressed. The virion } \\
\text { cannot replicate and is termed "replication-deficient". The two most } \\
\text { common methods are via: } \\
\text { 1) An adenovirus which utilizes double stranded DNA (dsDNA) } \\
\text { that is not integrated into the host genome. } \\
\text { A lentivirus makes use of RNA that is reverse transcribed into } \\
\text { DNA that is integrated into the host genome. }\end{array}$ \\
\hline Electroporation & $\begin{array}{l}\text { An electrical field is applied to cells through an electroporator that } \\
\text { contacts aluminum electrodes on each side of a cuvette containing the } \\
\text { cell suspension. Voltage, typically in the range of 1.0-1.5 kV, is applied } \\
\text { for a few microseconds up to a millisecond. This disturbs the cell } \\
\text { membrane and creates transient pores. The potential created can allow } \\
\text { charged molecules, such as DNA, to be driven through the pores }\end{array}$ \\
\hline Microinjection
\end{tabular}




\begin{tabular}{|c|l|}
\hline Sonoporation & $\begin{array}{l}\text { Inert gas microbubbles (MBs) are either oscillated or ruptured via } \\
\text { ultrasound near cell membranes creating microstreams and/or microjets } \\
\text { of fluid that increase the fluid shear stress. Like cell squeezing, the shear } \\
\text { stress creates transient pores in the cell membrane that increase } \\
\text { permeability }{ }^{[13]} \text {. A unique benefit of this technique is that while the } \\
\text { pores may seal rapidly, the increase in permeability lasts significantly } \\
\text { longer, up to 12 minutes }{ }^{[14]} \text { due to increases in endocytosis. }\end{array}$ \\
\hline
\end{tabular}

While some have seen approved clinical applications, such as the CAR T-cell cancer treatments detailed previously, each technique is plagued by challenges including, but not limited to, limited efficiency and consistency, for different reasons. Drawbacks of each technique are detailed in Table 2.

Table 2. Drawbacks of various transformation techniques.

\begin{tabular}{|c|c|}
\hline $\begin{array}{c}\text { Transformation } \\
\text { Technique }\end{array}$ & Drawbacks \\
\hline Viral Transduction & $\begin{array}{l}\text { - Virion size caps size of genetic material that can be introduced } \\
\text { - Gene can be randomly inserted - may lead to insertion } \\
\text { mutagenesis } \\
\text { - } \quad \text { Can be difficult to manufacture - classified as Biosafety Level } 2 \\
\text { - Can induce an immune system response } \\
\text { [27] }\end{array}$ \\
\hline Electroporation & 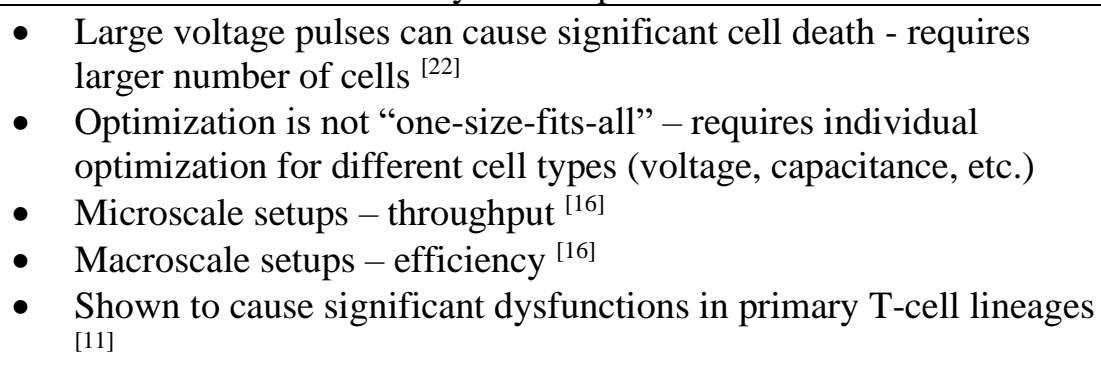 \\
\hline Microinjection & $\begin{array}{l}\text { Requires more complex equipment - microscope, } \\
\text { micromanipulators, micropipettes, etc. } \\
\text { - Difficult to scale up to treat large numbers of cells }{ }^{[4]}-\text { throughput }\end{array}$ \\
\hline Cell Squeezing & $\begin{array}{l}\text { - Small channels can clog easily } \\
\text { - DNA/RNA introduction is entirely passive (no driving force) - } \\
\text { efficiency } \\
\text { - Consistency of channel dimensions/geometry depending on } \\
\text { manufacturing method (ex. PDMS lithography) }\end{array}$ \\
\hline Sonoporation & $\begin{array}{l}\text { - } \text { Microscale setups - throughput } \\
\text { - Lower transformation efficiency compared to viral transduction }{ }^{[16]} \\
\text { - Newer - optimization necessary } \\
\text { - Acoustic waves affected by size and material of container - alters } \\
\text { bubble rupture }\end{array}$ \\
\hline
\end{tabular}




\section{Acoustofluidic Sonoporation}

Acoustofluidic sonoporation is a process in which cells, biologic(s), and microbubbles are passed through channels while ultrasound (US) is applied. This process has shown to significantly improve molecular delivery compared to bulk sonoporation alone. Previously, microfluidic channels (ex. 500 x $200 \mu \mathrm{m}$ ) were utilized, however the PDMS lithography production method resulted in significant geometrical inconsistencies between various channels. Newer iterations utilize 3D stereolithography (SLA) printing for increased control over channel dimensions, however, this has limited the minimum size of the channels to $1 \mathrm{~mm}$ x $1 \mathrm{~mm}$. Experimentation has shown that the larger channels can result in significant delivery of biologics and greater consistency as compared to the previous microfluidic channels. New research has demonstrated that the acoustic pressure generated by the ultrasound (US) application creates a pressure gradient within the fluidic channels that forces cells and biologics towards the opposite wall of the channel ${ }^{[5]}$, thereby combining the effects of cell squeezing and sonoporation. With this movement, larger channels (of the mm scale) may be able to achieve similar shear stresses and flow characteristics as microfluidic (of the $\mu \mathrm{m}$ scale) channels.

While the research and experimentation suggest the acoustofluidic sonoporation is effective in delivering biologics, there is lack of understanding of the physical forces that create differences in delivery between different channel geometries, namely size and shape. Cells are exposed to multiple types of stress, wall shear stress (WSS), fluid shear stress, and microbubble rupture shear stress, each of which have been shown to permeabilize cells. While studies have explored the shear stress components separately, the interactions of the various components have not been evaluated. There likely exists some relationship/correlation between the WSS and US/acoustic pressure, due to the movement of particles towards the opposite wall of application. This has significant implications on delivery efficiency and post-processing viability as Warboys et al. ${ }^{[29]}$ demonstrated that chronic (extended duration) shear exposure reduces cell permeability and inhibits post-processing mitosis which could not be reversed. These findings suggest some 
threshold in shear stress that cells can endure before the membrane cannot be repaired and dysfunction and/or death occur. Therefore, channel design is of critical importance in maximizing transformation efficacy without significantly compromising cell viability. Analyzing the fluid and particle flow in these acoustofluidic channels is necessary to develop the understanding that will drive optimization of channel design to maximize transformation efficiency.

This thesis aims to begin to fill this gap in understanding of the fluidic forces that particles experience during acoustofluidic sonoporation procedures. Outcomes of particular interest are the WSS generated by fluid flow, particle velocity and flow pattern(s), and particle residence time. The findings should assist with optimizing acoustofluidic sonoporation by developing a thorough understanding of the effects of channel design. Optimization is necessary to translate this technique of cell transformation into clinical practice, in which cell therapies derived from cell transformation (e.g. CAR T-cell Therapy) are becoming more popular and effective. 


\section{METHODS}

\section{SOLIDWORKS Modeling}

Three channel geometries were constructed in SOLIDWORKS 2019:

1) $1 \mathrm{~mm} \times 1 \mathrm{~mm} \times 25 \mathrm{~mm}$ Rectilinear channel

2) $2 \mathrm{~mm} \times 2 \mathrm{~mm} \times 25 \mathrm{~mm}$ Rectilinear channel

3) $1 \mathrm{~mm} \times 1 \mathrm{~mm}$ Concentric Spiral channel

Figures of each channel are shown in Figure 2.
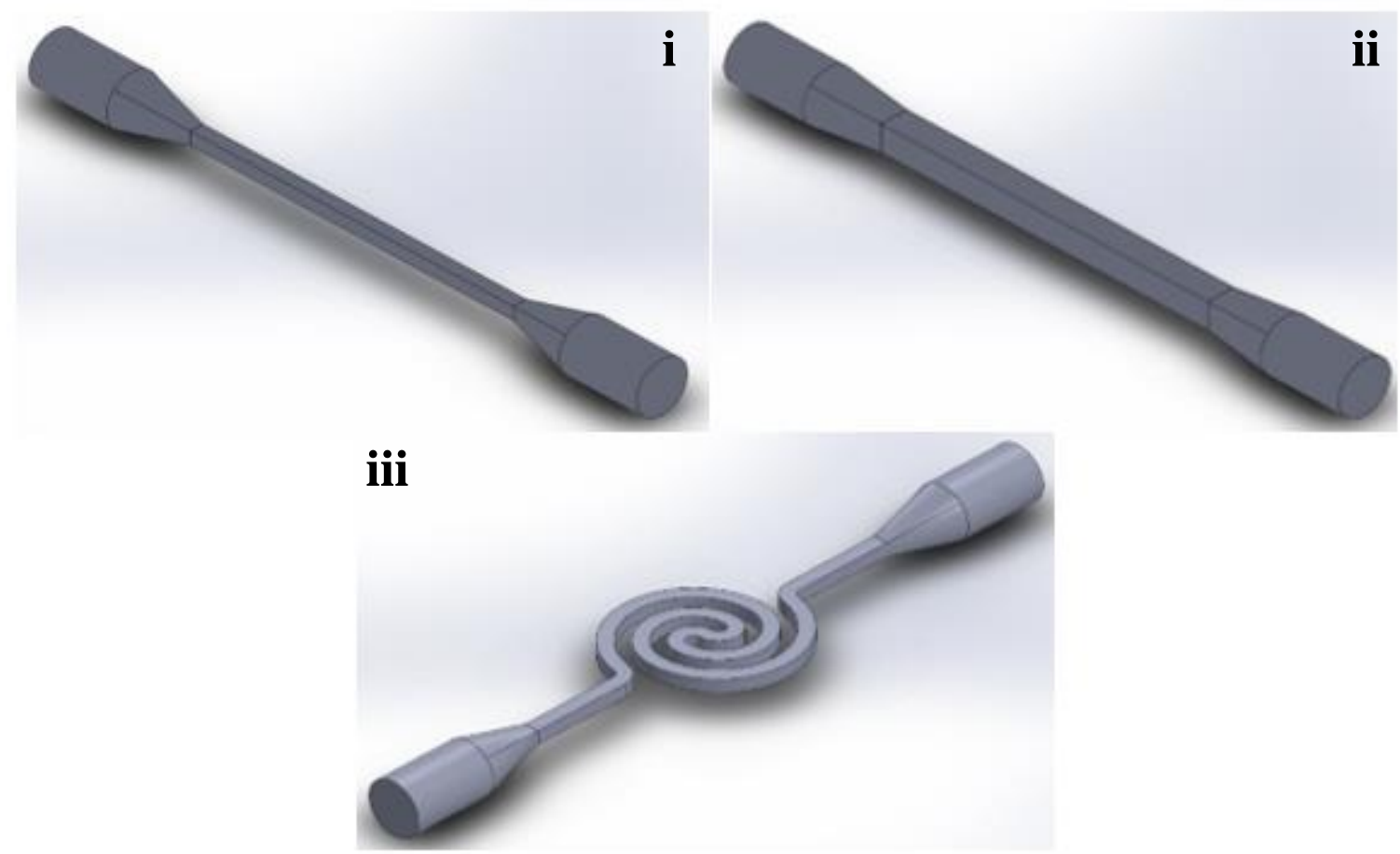

Figure 2. Renderings of the channels in SOLIDWORKS. Channels are defined by the size and pattern of the middle feature. i) $1 \mathrm{~mm}$ Rectilinear ii) $2 \mathrm{~mm}$ Rectilinear iii) $1 \mathrm{~mm}$ Concentric Spiral. Note: This numbering scheme will be utilized throughout.

To create each channel, the sketch of each was drawn on top plane and extruded to the appropriate height, $1 \mathrm{~mm}$ or $2 \mathrm{~mm}$. A plane was added $5 \mathrm{~mm}$ from each of the end faces of channel, with the normal pointing away from channel body. A center circle, with diameter of $3.97 \mathrm{~mm}$, was drawn on each plane and extruded for $6 \mathrm{~mm}$ from plane. This feature represents the body that can 
be connected to the pump (syringe or peristaltic). To connect the channel feature and inlet/outlet body features, a loft was created between the features with tangent faces and the result not being merged, which was critical for importing the parts into ANSYS.

\section{ANSYS Workbench Setup and Meshing}

A new 2020 ANSYS Academic Research Workbench project was created for each channel with the following components:
1) Geometry
2) Meshing
3) Fluent

The SOLIDWORKS models were imported into Geometry component. Using DesignModeler, each feature of the five features were named and Named Selections were defined including the inlet, outlet, and walls for each channel as shown in Figure 3. The resulting geometry data was transferred to the Meshing component.
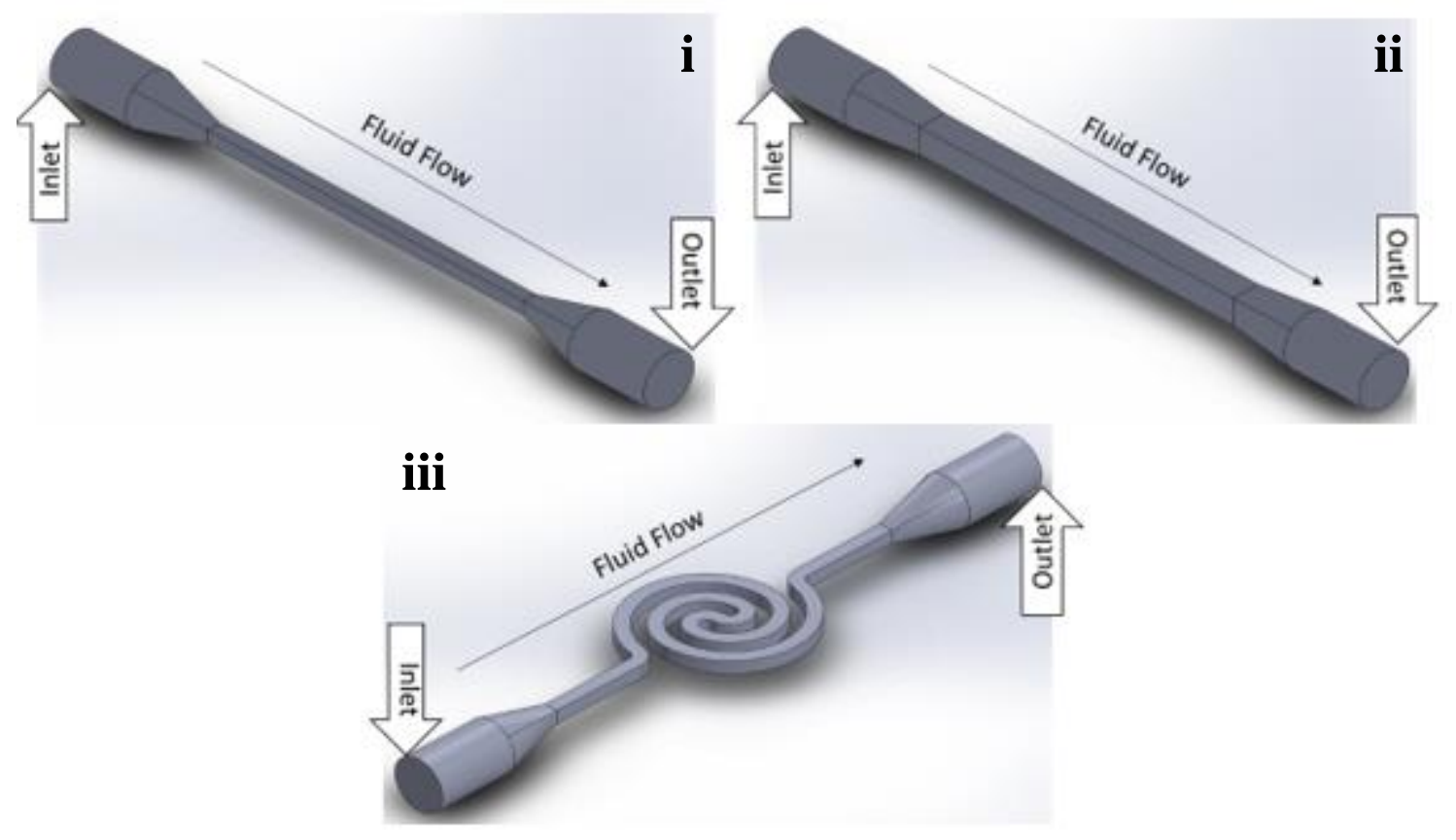

Figure 3. Labeling inlet, outlet, and fluid flow direction for each channel as was done in ANSYS DesignModeler. These orientations are preserved throughout the following analyses. 
Within the ANSYS Meshing component, bonded connections were created between successive features (i.e. Inlet_Body bonded to Inlet_Adapter, Inlet_Adapter bonded to Channel, Channel bonded to Outlet_Adapter, and Outlet_Adapter bonded to Outlet_Body) to ensure that ANSYS Fluent would register the entire channel as one system for flow simulations.

After creating the connections between the features, the Global Mesh settings were altered as follows. The Physics Preference was set to CFD with Fluent specified as the Solver Preference. The Element Size was kept at its default setting of $2.3667 \mathrm{~mm}$ but was set as an input parameter to test different mesh sizes for independence. Within the Sizing options, Adaptive Sizing and Capture Proximity were disabled, due to the relatively simple geometry of the channels, while both Mesh Defeaturing and Capture Curvature were enabled. All other Sizing settings were retained at the default values. To ensure that the mesh would produce accurate, reliable results, default settings were used within the Quality menu except for alterations as detailed; the Target Skewness was decreased to 0.7 , Smoothing was set to medium, and the Mesh Metric reported was skewness with the maximum, average, and standard deviation set as output parameters. Alongside the mesh independence analysis if any mesh size reported a maximum skewness $\geq 0.9$, the mesh size was excluded from further analysis. Due to the laminar flow scheme and low velocities to be analyzed, boundary/inflation layers were necessary to accurately represent flow near the walls, therefore, the following Inflation settings were utilized; Automatic Inflation was used within All Faces in Chosen Named Selection, with the selection specified as the walls (defined within DesignModeler previously). The method for the inflation was set to Smooth Transition and all settings were retained at the default value except for the Maximum Layers was decreased to four (4) and Collision Avoidance was changed to stair stepping.

A sweep method was added to the channel feature for both $1 \mathrm{~mm}$ channels and recorded as the first meshing step. This ensured the channel feature would be comprised of mostly hexahedral cells which have been shown to produce more accurate results with less computational time ${ }^{[20]}$. By recording the sweep method first, these cells would specify the type/shape of the cells at the 
interfaces with the Inlet/Outlet_Adapter features, and more generally, the entire channel volume. The mesh element size was set as an input parameter to align with the global mesh element size. The sweep method was not utilized in the $2 \mathrm{~mm}$ Rectilinear channel because of the limited size decrease from the Inlet/Outlet_Body to the Channel that prevented larger mesh sizes from being used. The face meshes on the inlet and outlet side could not match the volume mesh generated by the sweep method.

\section{ANSYS Fluent Analysis}

The mesh generated from the preceding steps was transferred to the Setup of the Fluent component. On startup, the following solver options were selected, Double Precision and Parallel Processing with sixteen (16) processes (CPU: AMD Ryzen 7 2700X Eight-Core Processor, 4.00 GHz). Within Fluent, the Energy, Viscous, and Discrete Phase models were enabled for the analysis. A laminar flow regime was selected for the fluid flow, as Ward et al. has shown that flow at low velocities in these small diameter channels is dominated by viscous forces resulting in low Reynolds numbers ${ }^{[30]}$. To confirm a laminar flow regime, the Reynolds number was calculated at four different regions for each channel as shown in Figure 4, with the threshold between laminar and turbulent flow set to 2300 .

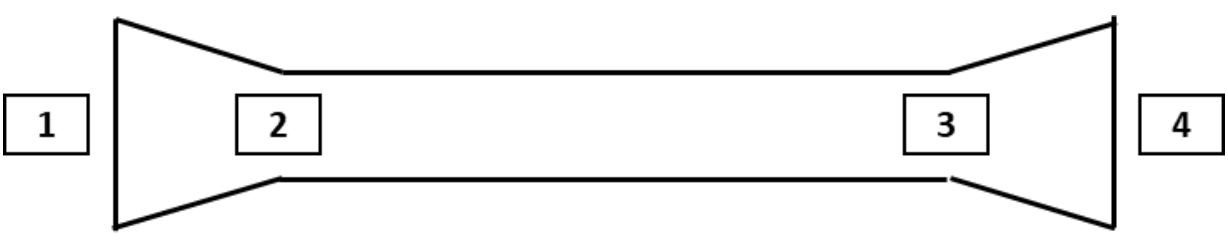

Figure 4. Simplified schematic of the fluidic channels with each region where the Reynolds number was calculated. Region 1 represents the inlet, Region 2 the beginning of the channel, Region 3 the end of the channel, and Region 4 the outlet.

The Reynolds number, $R e$, was calculated using Equation 1

$$
R e=\frac{D \rho V}{\mu}
$$


where $D$ is the diameter/height of the channel at each region, $\rho$ is the fluid density $\left(997 \mathrm{~kg} / \mathrm{m}^{3}\right.$ for liquid water), $V$ is the fluid velocity at each region, and $\mu$ is the dynamic viscosity $\left(0.00091 \mathrm{~N} * \mathrm{~s} / \mathrm{m}^{2}\right.$ for liquid water). The velocity, $V$, at region 2 was calculated using the continuity equation with an incompressible liquid as shown in Equation 2

$$
V_{1} A_{1}=V_{2} A_{2}
$$

where $A$ is the area of flow. An area-weighted average of velocity magnitude at the outlet surface was created and used to compute the velocity at region four (4) for each channel. Reynolds numbers were calculated for both Vin $=0.002$ and $0.01 \mathrm{~m} / \mathrm{s}$ with the results shown in Table 3 and 4 .

Table 3. Reynolds number calculations for each region of each channel at inlet velocity $=0.002 \mathrm{~m} / \mathrm{s}$. No region surpassed the $\mathbf{2 3 0 0}$ threshold, supporting the assumption of a laminar flow regime.

\begin{tabular}{|c|c|c|c|c|}
\hline \multicolumn{5}{|c|}{ Vin $=0.002 ~ \mathbf{~ m} / \mathbf{s}$} \\
\hline & Region 1 & Region 2 & Region 3 & Region 4 \\
\hline 1 mm Rectilinear & 8.6991 & 27.1241 & 27.1241 & 8.6802 \\
\hline $\mathbf{2}$ mm Rectilinear & 8.6991 & 13.5620 & 13.5620 & 8.7021 \\
\hline $\mathbf{1 ~} \mathbf{~ m m}$ Concentric Spiral & 8.6991 & 27.1241 & 27.1241 & 9.1929 \\
\hline
\end{tabular}

Table 4. Reynolds number calculations for each region of each channel at inlet velocity $=0.01 \mathrm{~m} / \mathrm{s}$. No region surpassed the $\mathbf{2 3 0 0}$ threshold, supporting the assumption of a laminar flow regime.

\begin{tabular}{|c|c|c|c|c|}
\hline \multicolumn{5}{|c|}{ Vin = 0.01 m/s } \\
\hline & Region 1 & Region 2 & Region 3 & Region 4 \\
\hline 1 mm Rectilinear & 43.4955 & 135.6203 & 135.6203 & 43.4922 \\
\hline 2 mm Rectilinear & 43.4955 & 67.8101 & 67.8101 & 43.5145 \\
\hline 1 mm Concentric Spiral & 43.4955 & 135.6203 & 135.6203 & 44.1079 \\
\hline
\end{tabular}

The discrete phase was set to interact with the continuous phase with the default DPM Iteration Interval, Maximum Number of Steps, and Step Length Factor. High-Res Tracking was enabled for increased accuracy of discrete phase variables. The Pressure Gradient Force was enabled within the Physical Models to account for any potential changes the inclusion of particles could have on the flow of the particles. Additional tracking options, including Accuracy Control, Track in Absolute Frame, and Linearize Source Terms were enabled. The Tracking Scheme was set to Automated with the default options retained. To complete the Discrete Phase model 
parameters, the Method was set to Hybrid and Use DPM Domain was selected to reduce the computational load required.

One (1) injection was added to the discrete phase analysis. Perfluorobutane $\left(\mathrm{C}_{4} \mathrm{~F}_{10}\right)$, which comprises the microbubbles used within the sonoporation procedures, was added as an inert particle material with the following properties ${ }^{[19]}$ :

1) Density: $24.6 \mathrm{~kg} / \mathrm{m}^{3}$

2) Specific Heat: $809 \mathrm{~J} /(\mathrm{kg} * \mathrm{~K})$

The Injection Type was changed to surface, and the inlet was selected as the release surface. A Rosin-Rammler diameter distribution was used with a minimum diameter of $1 \mathrm{e}^{-06}$, a maximum diameter of $3 \mathrm{e}^{-06} \mathrm{~m}$, and a mean diameter of $2 \mathrm{e}^{-06} \mathrm{~m}$. The Spread Parameter and Number of Diameters were retained at the default values. The options to Scale Flow Rate by Face Area and Inject Using Face Normal Direction were enabled. The Velocity Magnitude was set to the "Velocity_Inlet" (discussed later), as the particles are suspended in the injected fluid. An input parameter defined as "Microbubble_Flow_Rate" was specified for the Total Flow Rate to allow for exploration of particle transport at various concentrations (default value: $1 \mathrm{e}^{-20} \mathrm{~kg} / \mathrm{s}$ ). Additionally, within the Physical Models options, the Drag Law was kept as spherical and rotation was enabled following the Dennis-et-al Rotational Drag Law; however, the Angular Velocity Magnitude was set to $0 \mathrm{rad} / \mathrm{s}$ to decrease the computational load of each simulation.

Water-liquid $\left(\mathrm{H}_{2} \mathrm{O}\right)$, with default properties, was added as a fluid material and set for both interior cell zones. While phosphate buffered saline (PBS) is the typical fluid that cells are suspended in during acoustofluidic sonoporation, its density $(1.01 \mathrm{~g} / \mathrm{mL})$ is only $1 \%$ different and its viscosity $(0.8882 \mathrm{cP})$ is only $2.45 \%$ from liquid water at $25^{\circ} \mathrm{C}$. Therefore, simulating flow with liquid water should only produce negligible differences during calculations. For the inlet boundary, two input parameters were created, "Velocity_Inlet" and "Pressure_Inlet", for the Velocity Magnitude and Supersonic/Initial Gauge Pressure settings, respectively. This allows for the 
exploration of the fluid dynamics at various flow rates and pressures. The outlet boundary pressure was set to $101325 \mathrm{~Pa}(1 \mathrm{~atm})$ and kept constant.

Residuals were monitored at the default values except for continuity, which was decreased to $1 \mathrm{e}^{-05}$ to ensure solutions reached a steady-state/converged. The following Report Definitions were created to analyze the simulation results:

1. Volume Average of Velocity Magnitude

2. Max of Velocity Magnitude

3. Volume Average of Static Pressure

4. Max of Static Pressure

5. Volume Average of Wall Shear Stress (WSS)

6. Area-Weighted Average of Wall Shear Stress (WSS)

7. Area-Weighted Average of Velocity Magnitude, at the outlet only to calculate the Reynolds number of the flow

The default initialization scheme was utilized (Hybrid for ten (10) iterations). The Time Scale Factor was retained at one (1) and the Number of Iterations was increased to eight hundred (800) to ensure that solutions would converge to a steady state.

After initializing the simulation, several graphics were created to visually interpret the results including:

1. A faces-only Mesh, excluding the interior of the channel

2. A WSS Contour

3. A static pressure Contour

4. A velocity magnitude Contour, interior portions only

5. Pathlines of velocity magnitude, interior portions only

6. A Particle Track of particle residence time, interior portions only

7. A Particle Track of particle velocity magnitude, interior portions only 
8. A Scene of the velocity pathlines in the faces-only mesh (set to $70 \%$ transparency)

9. A Scene of the particle residence time particle tracks in the faces-only mesh (set to $75 \%$ transparency)

10. A Scene of the particle velocity magnitude particle tracks in the faces-only mesh (set to $75 \%$ transparency)

A mesh independence analysis was performed for each channel to determine the optimal mesh. Mesh element sizes from $0.2 \mathrm{~mm}$ to $3 \mathrm{~mm}$ were analyzed for each channel. Results of the analyses are shown in Figure 5. It should be noted that $0.2 \mathrm{~mm}$ was the smallest mesh element size that could be tested due to the limited CPU power of the computer used and the 512,000-cell cap imposed by the Academic Research version of ANSYS Fluent.
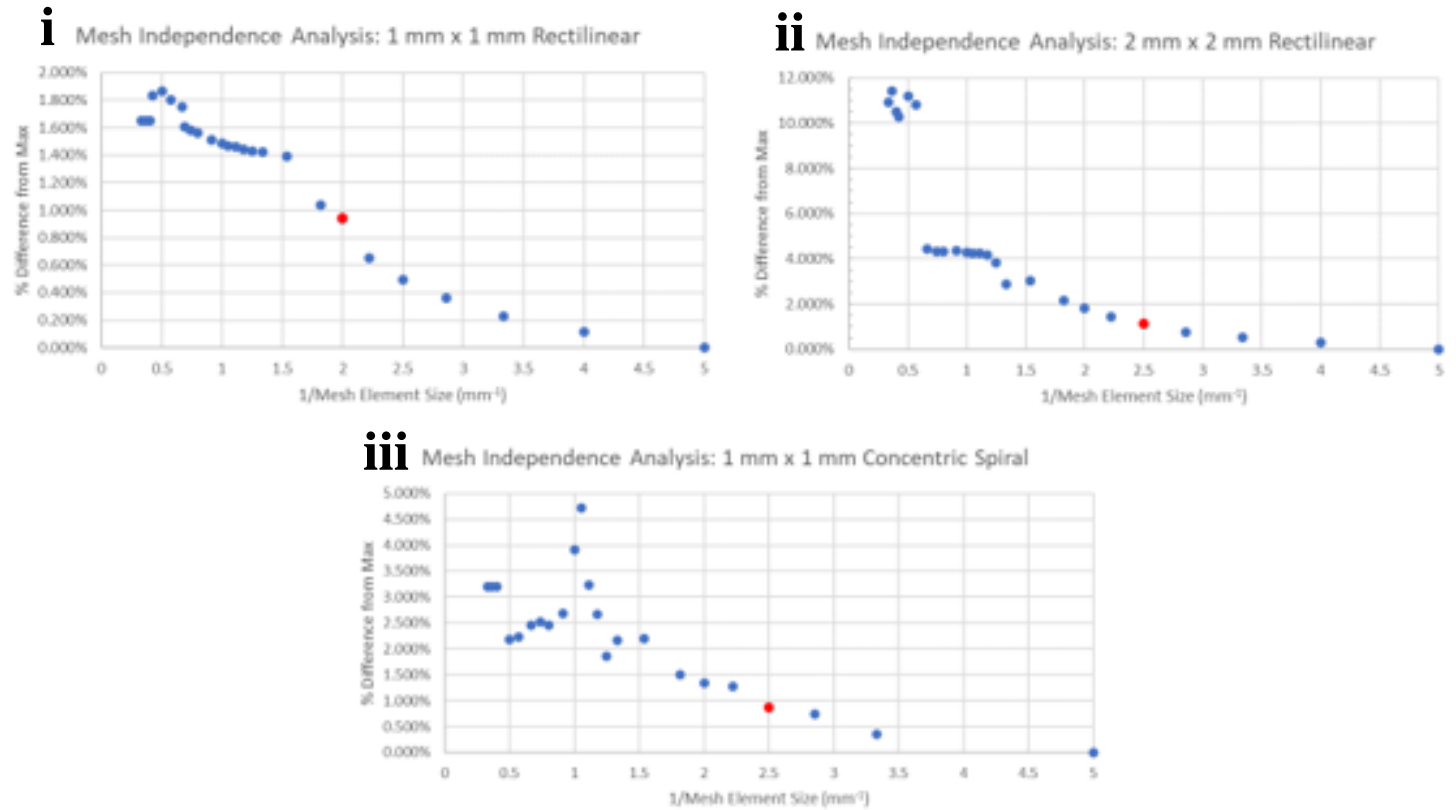

Figure 5. Mesh independence analyses for all the channel geometries. The smallest mesh size tested, $0.2 \mathrm{~mm}$, produced the maximum volume average velocity and a percent difference was calculated for the other mesh sizes. The largest mesh that had an $\sim 1 \%$ difference was selected for each channel and is represented by the red dot. 
The statistics for each channel at the selected/optimal mesh are given in Table 5.

Table 5. Selected characteristics for each channel at the optimal mesh size.

\begin{tabular}{|c|c|c|c|c|c|c|c|}
\hline Channel & $\begin{array}{c}\text { Mesh } \\
\text { Element } \\
\text { Size } \\
(\mathbf{m m})\end{array}$ & Nodes & Elements & $\begin{array}{c}\text { Max } \\
\text { Skewness }\end{array}$ & $\begin{array}{c}\text { Average } \\
\text { Skewness }\end{array}$ & $\begin{array}{c}\text { Skewness } \\
\text { Standard } \\
\text { Deviation }\end{array}$ & $\begin{array}{c}\text { \% } \\
\text { Difference }\end{array}$ \\
\hline $\begin{array}{c}\mathbf{1} \text { mm } \\
\text { Rectilinear }\end{array}$ & 0.5 & 7227 & 18169 & 0.77631 & 0.23433 & 0.12624 & $0.938 \%$ \\
\hline $\begin{array}{c}\mathbf{2} \text { mm } \\
\text { Rectilinear }\end{array}$ & 0.4 & 18675 & 49495 & 0.69371 & 0.25546 & 0.13559 & $1.101 \%$ \\
\hline $\begin{array}{c}\mathbf{1} \text { mm } \\
\text { Concentric } \\
\text { Spiral }\end{array}$ & 0.4 & 13784 & 30690 & 0.71346 & 0.20709 & 0.12755 & $0.860 \%$ \\
\hline
\end{tabular}

Jurkat T-Cell Culturing, Transfection, and Analysis

Jurkat T-cells were diluted to a concentration of $100,000 / \mathrm{mL}$ in $18 \mathrm{~mL}$ of supplemented RPMI medium which contains RPMI medium, $10 \%$ fetal bovine serum, and $2 \%$ penicillin/streptomycin. The cell solution was divided into 3-mL aliquots. Seven (7) experimental groups were determined with three (3) replications per group ( $n=3$ /group):

1. Group \#0: Cells only without calcein

2. Group \#1: No flow, no ultrasound

3. Group \#2: Flow through $1 \mathrm{~mm}$ Rectilinear channel without ultrasound

4. Group \#3: Flow through $2 \mathrm{~mm}$ Rectilinear channel without ultrasound

5. Group \#4: Flow through $1 \mathrm{~mm}$ Rectilinear channel with ultrasound

6. Group \#5: Flow through $2 \mathrm{~mm}$ Rectilinear channel with ultrasound

7. Group \#6: Flow through $1 \mathrm{~mm}$ Rectilinear channel with ultrasound. Samples were passed through the device twice to double the ultrasound exposure time.

For groups \#1-6, $333 \mu \mathrm{L}$ of $1 \mathrm{mg} / \mathrm{ml}$ calcein solution was added to each 3-mL cell sample. For groups \#2-6, flow was through the specified 3D-printed acoustofluidic device using a peristaltic pump at $1.5 \mathrm{~mL} / \mathrm{min}$. For groups \#4-6, $10 \mu \mathrm{L} / \mathrm{mL}$ of cationic microbubbles, synthesized using the procedure described within Center et al. ${ }^{[8]}$, were added to the solution and ultrasound was applied 
using a Verasonics P4-1 transducer placed directly on the device at an output peak negative pressure of 3.8 MPa, as shown in Figure 6.

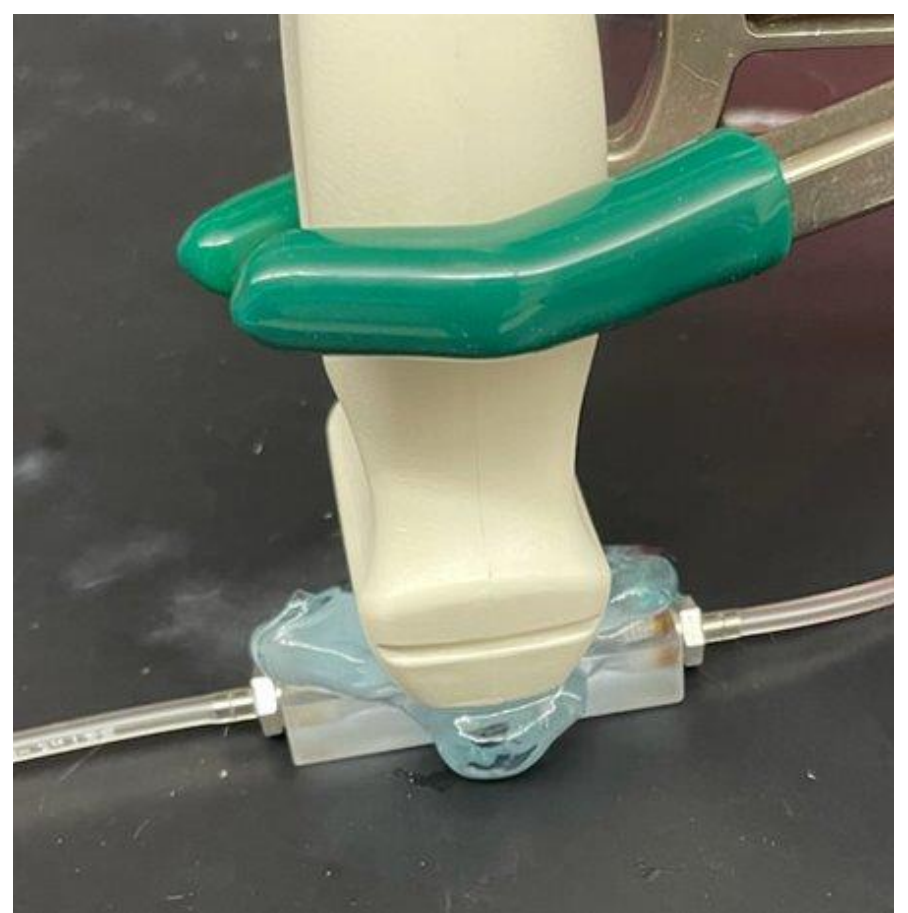

Figure 6. Image of acoustofluidic sonoporation setup with the channel connected at both the inlet and outlet and the Verasonics US transducer placed on the device.

After the treatment, all samples were washed 3x using the following protocol:

1. Centrifuge at $1500 \mathrm{~g}$ for $5 \mathrm{~min}$

2. Aspirate $0.9 \mathrm{~mL}$ of supernatant, then add $0.9 \mathrm{ml}$ of $\mathrm{PBS}$ and resuspend pellet

3. Centrifuge at $1500 \mathrm{~g}$ for $5 \mathrm{~min}$

4. Aspirate $0.9 \mathrm{~mL}$ of supernatant, then add $0.9 \mathrm{ml}$ of PBS and resuspend pellet

5. Centrifuge again at $1500 \mathrm{~g}$ for $5 \mathrm{~min}$

6. Aspirate $0.9 \mathrm{~mL}$ of supernatant, then add $250 \mu \mathrm{L}$ of PBS and resuspend pellet

A $0.5 \mathrm{mg} / \mathrm{mL}$ propidium iodide (PI) solution was prepared and after washing, $5 \mu \mathrm{L}$ of the PI solution was added to each sample producing a final concentration in each sample of $10 \mu \mathrm{g} / \mathrm{mL}$.

All samples were then run through flow cytometry analysis using a MACSQuant Analyzer 10 
(Miltenyi Biotec, Auburn, CA, USA) with 10,000 events recorded per sample. Data was analyzed using FlowJo (Ashland, OR, USA) to determine green calcein fluorescence (in the B1 "FITC channel), which is an indicator of delivery efficiency, and red PI fluorescence (in the B3 "PerCP" channel), which is an indicator of cell viability. The data from this experiment was analyzed using an analysis of variance (ANOVA) in Minitab 19 with channel, flow condition, US inclusion, and number of flow throughs included as factors. The significance level was set to $\alpha=0.05$.

For a second set of biologic delivery cell experiments, that same procedure described above was utilized with the following experimental groups ( $n=6 /$ group):

1. Group \#1: Flow through $1 \mathrm{~mm}$ Rectilinear channel without ultrasound

2. Group \#2: Flow through $2 \mathrm{~mm}$ Rectilinear channel without ultrasound

3. Group \#3: Flow through $1 \mathrm{~mm}$ Concentric Spiral channel without ultrasound

4. Group \#4: Flow through $1 \mathrm{~mm}$ Rectilinear channel with ultrasound

5. Group \#5: Flow through $2 \mathrm{~mm}$ Rectilinear channel with ultrasound

6. Group \#6: Flow through $1 \mathrm{~mm}$ Concentric Spiral channel with ultrasound

Unlike experiment one above, the data from this second experiment was analyzed using student t-test comparisons, with the Bonferroni correction, in Minitab 19. The significance level was set to $\alpha=0.05$ for all tests.

These two experiments were completed in order to compare delivery results to the CFD outputs to potentially correlate certain variables (e.g. WSS) with differences in delivery. While exact causes for variations in delivery cannot be determined without further research, the results should suggest areas of optimization, additional variables to explore, and constraints of current technology and techniques. This work provides the beginnings of a foundation from which to develop greater understanding about the causes of cell permeabilization during acoustofluidic sonoporation procedures. 


\section{RESULTS/DISCUSSION}

\section{ANSYS Computational Fluid Dynamics (“CFD”) Output}

The first outcome of interest was wall shear stress (WSS), as this is one of the most critical factors in cell transformation/biologic delivery. WSS contours were created for each channel and scaled to a common range; the minimum value was set to $2.32 \mathrm{e}^{-03} \mathrm{~Pa}$ and the maximum value was set to $3.29 \mathrm{e}^{-01} \mathrm{~Pa}$. The results for each channel are shown in Figure 7 .
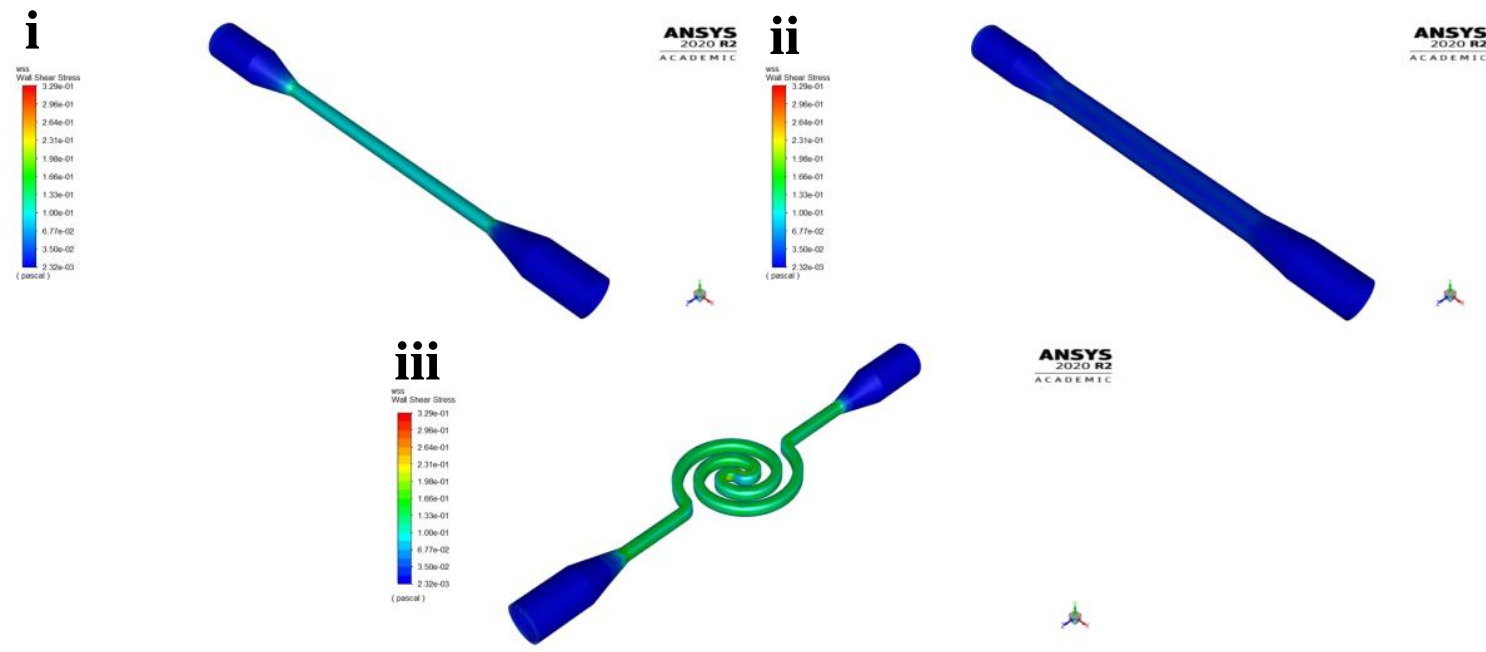

ANSYS
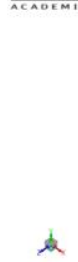

Figure 7. Scaled WSS contours for each channel geometry. In all channels, the maximum occurs within Channel feature, with the $1 \mathrm{~mm}$ Concentric Spiral producing the maximum value. Due to the maximum scale value being based on the $1 \mathrm{~mm}$ Concentric Spiral, differences in the $2 \mathbf{~ m m}$ Rectilinear channel are not easily distinguishable.

As shown in Figure 7, the $1 \mathrm{~mm}$ Concentric Spiral channel produces greater WSS throughout the channel, with peak values occurring near the center of the channel at the inner edges of the $180^{\circ}$ turn. However, in the $1 \mathrm{~mm}$ and $2 \mathrm{~mm}$ Rectilinear channels, the peak WSS occurs where the lofted section from the inlet and outlet joins with the channel. To compare the WSS generated by each channel, the volume average WSS output parameter was investigated for each channel geometry and the minimum and maximum inlet velocity values, the results are shown in Figure 8 and Table 6. 


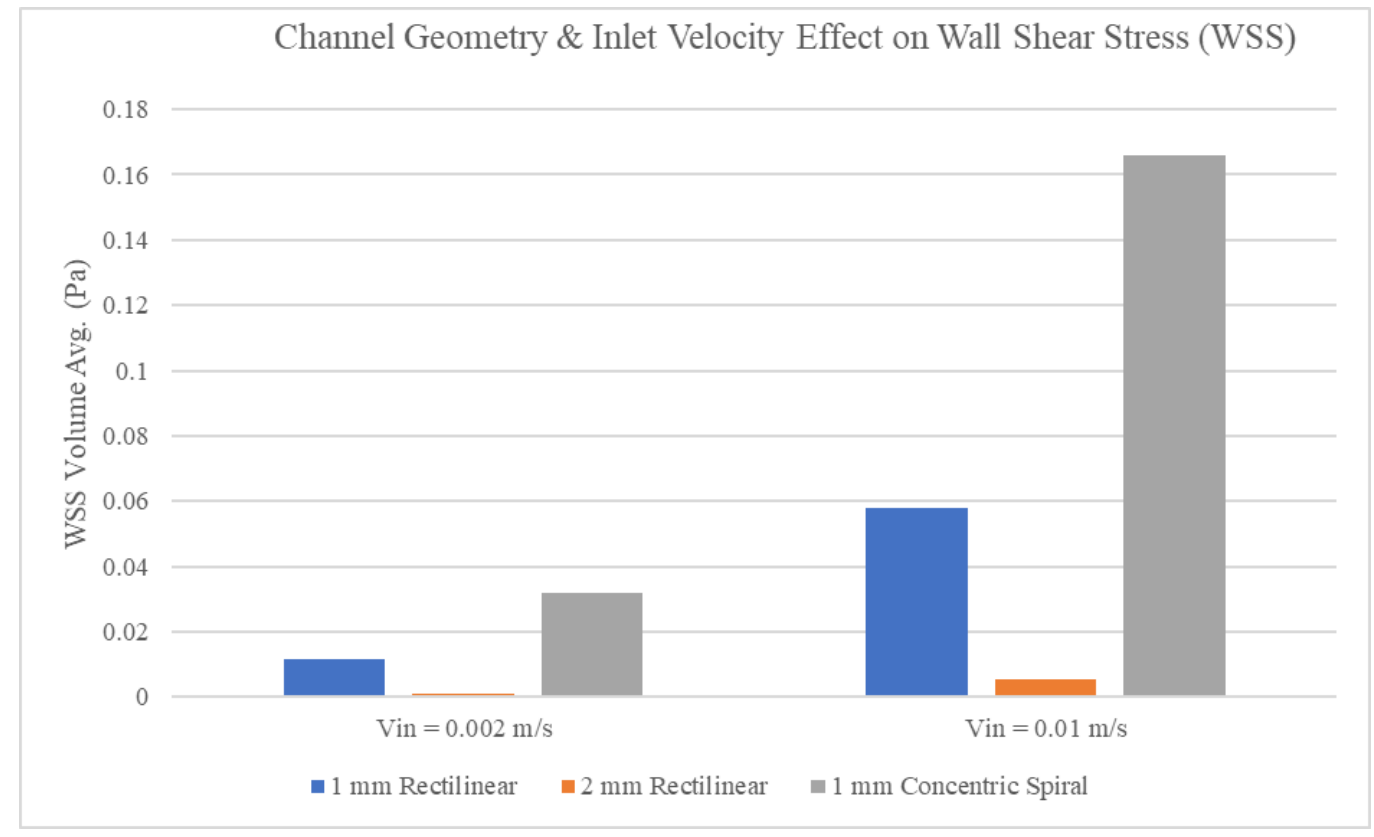

Figure 8. Comparison of volume average WSS for each channel geometry at two different inlet velocities (Vin) -0.002 and $0.01 \mathrm{~m} / \mathrm{s}$. In both cases, the $1 \mathrm{~mm}$ Concentric Spiral channel produced the greatest value while the $2 \mathrm{~mm}$ Rectilinear channel produced the smallest.

Table 6. Volume average WSS values at each inlet velocity.

\begin{tabular}{|c|c|c|}
\hline \multirow{2}{*}{ Channel } & Vin $=\mathbf{0 . 0 0 2} \mathbf{~ m} / \mathbf{s}$ & Vin $=\mathbf{0 . 0 1} \mathbf{~ m} / \mathbf{s}$ \\
\cline { 2 - 3 } & WSS Vol Avg. (Pa) & WSS Vol Avg. (Pa) \\
\hline 1 mm Rectilinear & 0.01151 & 0.05808 \\
\hline 2 mm Rectilinear & 0.00110 & 0.00554 \\
\hline 1 mm Concentric Spiral & 0.03183 & 0.16574 \\
\hline
\end{tabular}

The WSS output parameter calculations support the results demonstrated within the WSS contours, with the $1 \mathrm{~mm}$ Concentric Spiral channel producing the maximum WSS. This is likely caused by the fact fluid at the inner edge of each curve/turn flows faster than fluid at the outer edge. The regional increase in flow rate proportionately increases the WSS. It should be noted that when the inlet velocity is set to $0.002 \mathrm{~m} / \mathrm{s}$, the $1 \mathrm{~mm}$ Rectilinear channel is $946.36 \%$ greater than the 2 $\mathrm{mm}$ Rectilinear channel and the $1 \mathrm{~mm}$ Concentric Spiral channel is $176.54 \%$ greater than the $1 \mathrm{~mm}$ Rectilinear. This same trend exists when the inlet velocity is increased by $5 \mathrm{x}$, however, the percent difference increases slightly between the $1 \mathrm{~mm}$ and $2 \mathrm{~mm}$ Rectilinear channels to $948.38 \%$ (a 2.02\% 
increase) and between the $1 \mathrm{~mm}$ Concentric Spiral and $1 \mathrm{~mm}$ Rectilinear to $185.37 \%$ (a 8.83\% increase).

Additionally, the WSS was investigated at two different inlet pressures, 1 and 2 atm, with results shown in Figure 9 and Table 7.

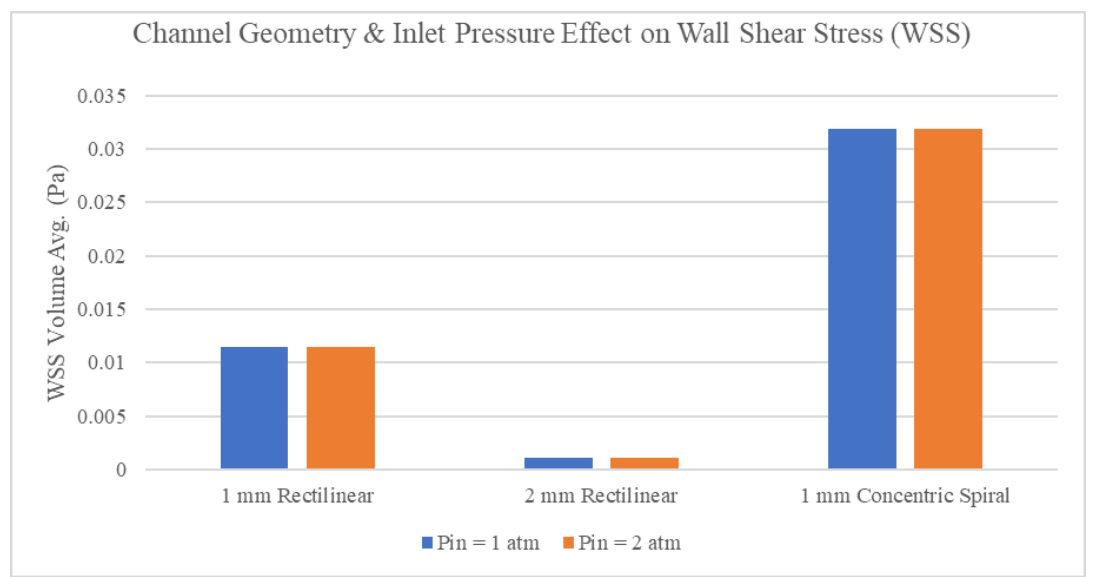

Figure 9. Comparison of volume average WSS output for each channel at both inlet pressure $=1$ and $2 \mathrm{~atm}$. For all three geometries, there is a negligible $(<1 \%)$ difference between the calculated values. This demonstrates that fluid pressure likely does not have a significant effect WSS particles experience.

Table 7. Volume average WSS values at each inlet pressure.

\begin{tabular}{|c|c|c|}
\hline \multirow{2}{*}{ Channel } & Pin = 1 atm & Pin = 2 atm \\
\cline { 2 - 3 } & WSS Vol Avg. (Pa) & WSS Vol Avg. (Pa) \\
\hline 1 mm Rectilinear & 0.01151 & 0.01151 \\
\hline 2 mm Rectilinear & 0.00110 & 0.00110 \\
\hline 1 mm Concentric Spiral & 0.03183 & 0.03183 \\
\hline
\end{tabular}

Analysis of the WSS measurements in Table 7 highlights that there is nearly no difference in WSS values when the inlet pressure is increased. This is likely due to how WSS, $\tau$, is calculated in a laminar flow scheme, as defined by Equation 3

$$
\tau=-\frac{3 \mu Q}{2 W h^{2}}
$$

where $\mu$ is dynamic viscosity, $Q$ is the flow rate, $W$ is the width of the channel, and $h$ is the height of the channel. Pressure is not one of the variables, nor influences any of the variables as $\mu$ is 
characteristic of the fluid which is assumed to be incompressible and therefore constant, $Q$ is constant value set on the pump (syringe or peristaltic), and $W$ and $h$ are the geometric dimensions of the channel.

Further analysis of the WSS at each inlet velocity tested demonstrates a unique trend for each geometry, as shown in Figure 10. As the inlet velocity value increases, the difference in WSS generated by each channel diverges by greater amounts. A possible explanation for this is the location in which particles flow through each geometry, which is discussed in more detail later. Briefly, in the Rectilinear channels, particles seem more distributed throughout the channel volume but in the spiral channel, particles tend to concentrate near the turns. As mentioned previously, higher flow rates at the inner edge of each curve/turn increases WSS, as highlighted in Equation 3. Additionally, this is likely influenced by the flow regime becoming less laminar with everincreasing fluid velocity that results in swirling/mixing, which should produce more interaction with the walls of the channel.

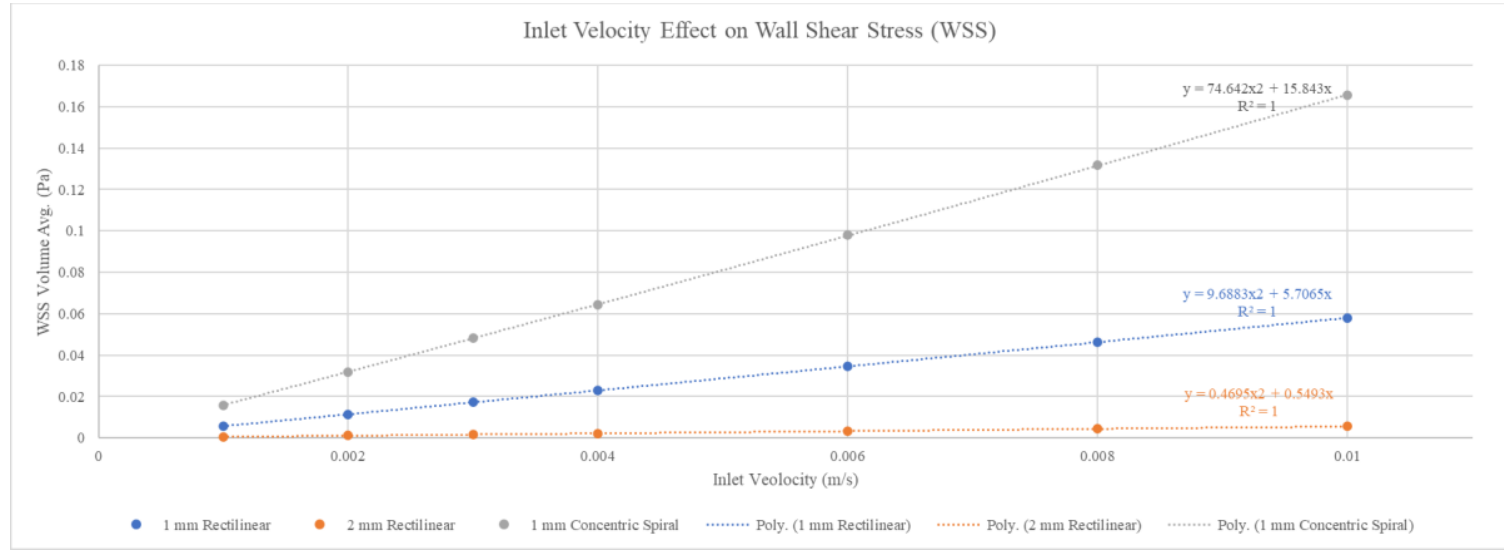

Figure 10. Comparison of WSS generated in each channel geometry at various inlet velocities. A unique trend exists in that as the inlet velocity increases, the difference in WSS generated by each channel increases. This difference could be critical in optimizing channel geometry to maximize biologic delivery and minimize decrease in cell viability for acoustofluidic sonoporation procedures.

It is important to consider the proportion of particles that are experiencing WSS, as not every particle can flow fully along the walls of each channel. Jurkat T-cells typically have a diameter between 5 and $7 \mu \mathrm{m}$, so in a $1 \mathrm{~mm}$ diameter channel, anywhere between $\sim 142$ and 200 
cells could physically fit across the width of the channel. Only those immediately adjacent to the wall will experience significant WSS, which may influence biologic delivery/cell transformation. A DPM Concentration contour was created on the mid-plane in the $\mathrm{Z}$ axis to analyze the spread of particles in each channel to see a relative distribution of particles that likely experience high WSS values. These results illustrate that particle concentrations may not be uniform between the different sides of the Concentric Spiral geometry, as compared to both Rectilinear geometries. It should be noted that the particles analyzed here were the perfluorobutane microbubbles and not specifically cells, but these results provide some insight into particle movement and distribution throughout each geometry given the expected interactions between cells and microbubbles during acoustofluidic sonoporation procedures. The contours, specifically focused on the Channel feature, are shown in Figure 11 with a common scale of $0 \mathrm{~kg} / \mathrm{m}^{3}$ to $7.75 \mathrm{e}^{-13} \mathrm{~kg} / \mathrm{m}^{3}$.

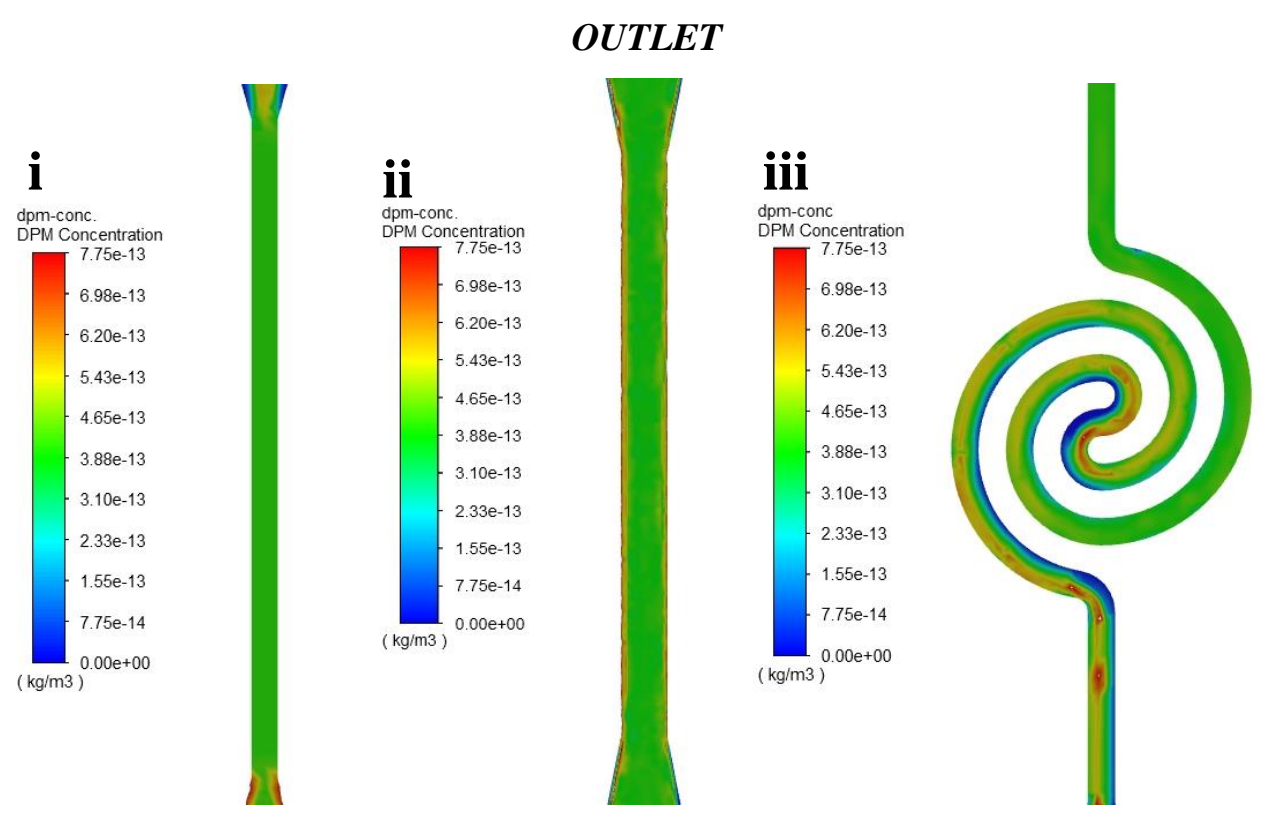

INLET

Figure 11. DPM Concentration contours for each of the channel geometries analyzed. In both Rectilinear channels, the spread is fairly uniform throughout the area. In the $\mathbf{2} \mathbf{~ m m}$ Rectilinear channel, there does appear to be a slightly higher concentration of particles near the walls. In the 1 mm Concentric Spiral, the distribution of particles is not uniform. A higher concentration tends to exist near the walls after the inlet and continuing until after the $180^{\circ}$ turn, which may suggest that a greater percentage of particles are experiencing a higher WSS in this channel compared to the Rectilinear geometries. 
To explore how fluid flows through each channel, a pathlines graphic was created of the velocity magnitude for each channel and scaled to a common range; the minimum value was set to $0 \mathrm{~m} / \mathrm{s}$ and the maximum value was set to $3.77 \mathrm{e}^{-02} \mathrm{~m} / \mathrm{s}$. The results for each channel are shown in Figure 12.

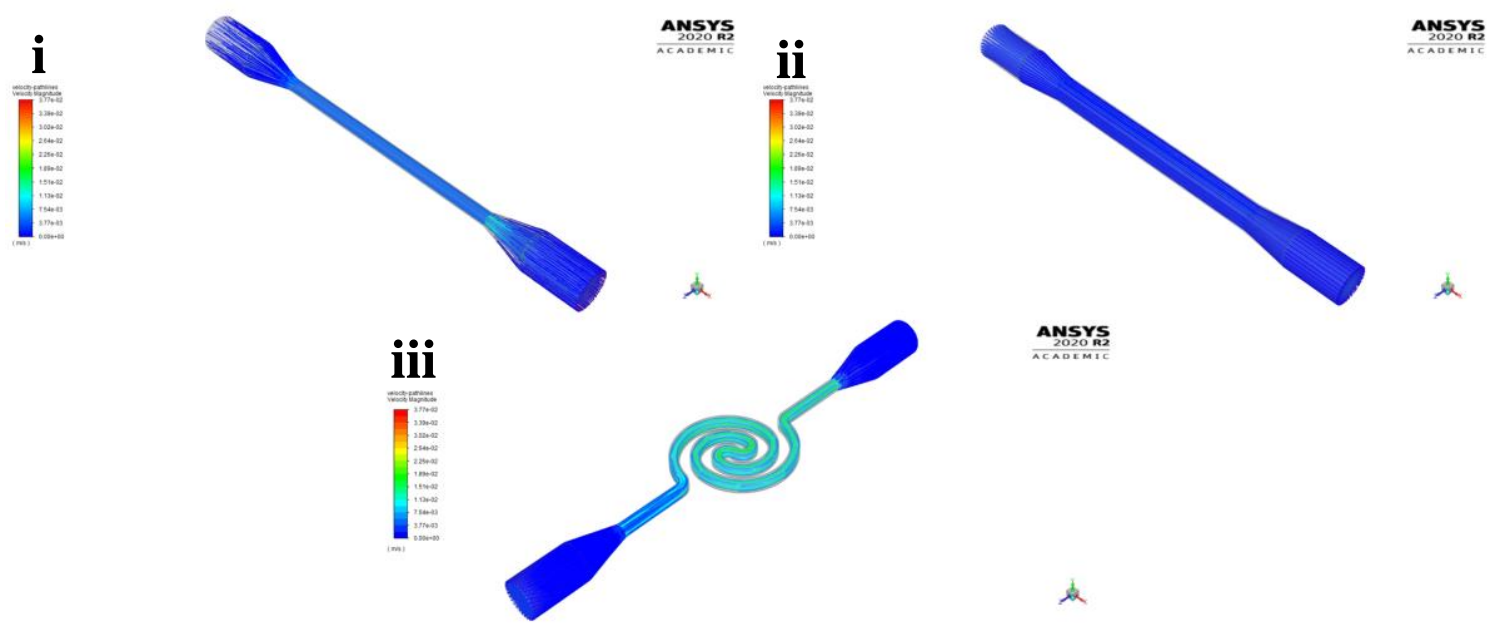

Figure 12. Scaled velocity pathline contours for each channel geometry. For the $\mathbf{1} \mathbf{m m}$ Rectilinear channel, the peak occurs at the connection between the channel and outlet loft, whereas in the $1 \mathbf{~ m m}$ Concentric Spiral, the peak occurs after the first curve and continues until the outlet loft.

As shown in Figure 12, the $1 \mathrm{~mm}$ Concentric Spiral channel produces greater velocity throughout the channel, with the peak value(s) occurring following the first turn and continuing until the channel connects to the loft. In the $1 \mathrm{~mm}$ Rectilinear channel, the peak velocity occurs where the lofted section from the inlet and outlet joins with the channel. This is explained through the continuity equation which states that to maintain a constant flow rate in a smaller area, the velocity must increase when the fluid is incompressible. The agreement between established defining fluid dynamic equations and characteristics with the CFD output supports the validity of these results. To quantitatively compare the velocity profiles of each channel, the volume average velocity magnitude output was investigated at both the minimum and maximum inlet velocity values; the results are shown in Figure 13 and Table 8. 


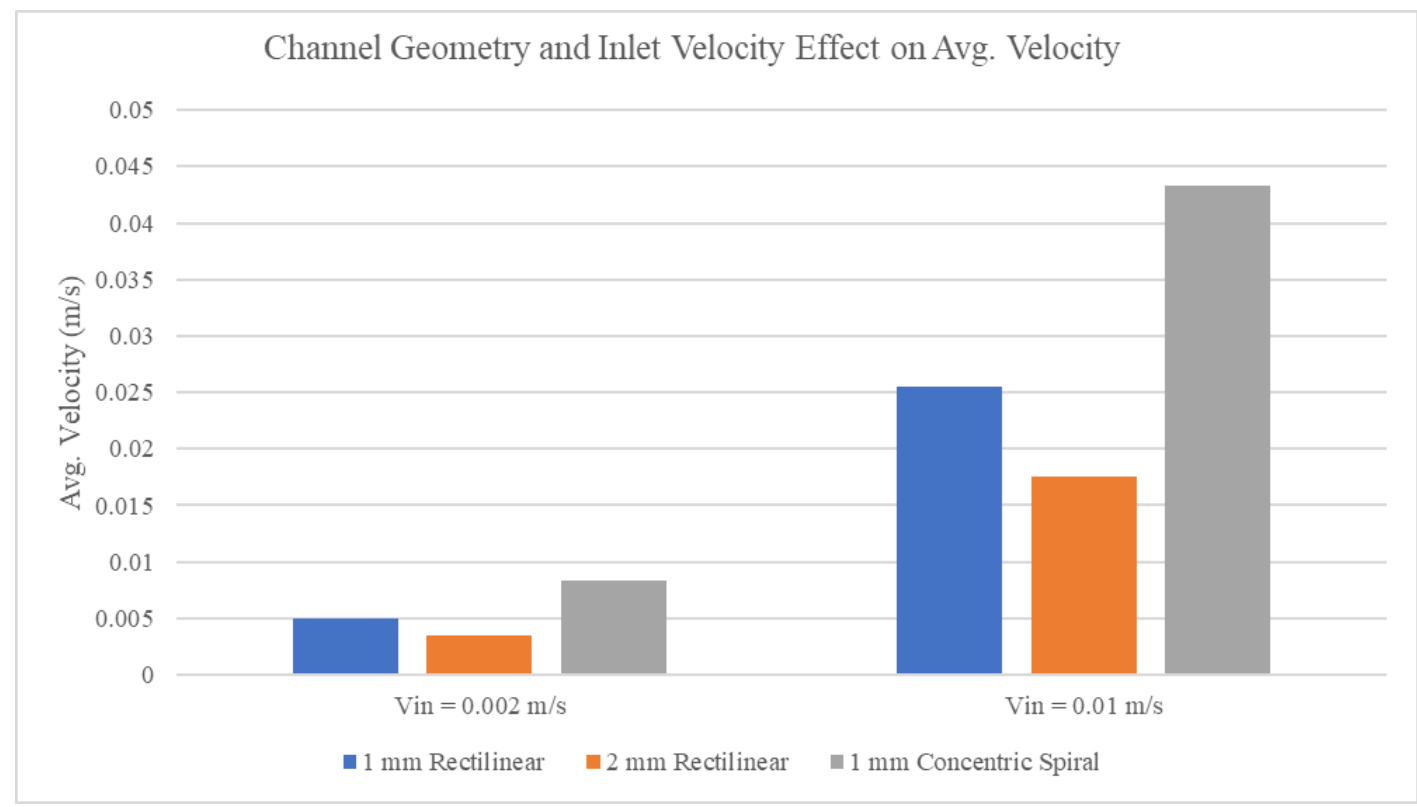

Figure 13. Comparison of volume average velocity output for each channel at both Vin $=0.002$ and $0.01 \mathrm{~m} / \mathrm{s}$. Similar to the WSS values highlighted in Figure 8, the $1 \mathrm{~mm}$ Concentric Spiral generates the greatest value, and the values diverge by greater amounts as Vin increases.

Table 8. Volume average velocity values at different inlet velocity values

\begin{tabular}{|c|c|c|}
\hline \multirow{2}{*}{ Channel } & Vin $=\mathbf{0 . 0 0 2} \mathbf{~ m} / \mathbf{s}$ & Vin $=\mathbf{0 . 0 1} \mathbf{~ m} / \mathbf{s}$ \\
\cline { 2 - 3 } & Velocity Avg. $(\mathbf{m} / \mathbf{s})$ & Velocity Avg. $(\mathbf{m} / \mathbf{s})$ \\
\hline 1 mm Rectilinear & 0.00501 & 0.02549 \\
\hline 2 mm Rectilinear & 0.00351 & 0.01757 \\
\hline 1 mm Concentric Spiral & 0.00828 & 0.04329 \\
\hline
\end{tabular}

As shown within Table 8, a 5x increase in inlet velocity resulted in an approximately 5x increase in resultant average velocity. Similar to the WSS trend shown in Figure 8, as the inlet velocity increases, the separation in average velocity increases. At Vin $=0.002 \mathrm{~m} / \mathrm{s}$, the percent difference between the $1 \mathrm{~mm}$ Rectilinear Channel and $1 \mathrm{~mm}$ Concentric Spiral is $65.27 \%$ versus at Vin $=0.01 \mathrm{~m} / \mathrm{s}$, the percent difference is $69.83 \%$ (a $4.56 \%$ increase). Between the $1-$ and 2-mm Rectilinear channels, the difference increases by $2.34 \%$, from $42.73 \%$ at Vin $=0.002 \mathrm{~m} / \mathrm{s}$ to $45.08 \%$ at Vin $=0.01 \mathrm{~m} / \mathrm{s}$. Combining the results for both WSS and average velocity, a pattern arises: changes to flow within the $1 \mathrm{~mm}$ Concentric Spiral geometry have a greater impact on the 
fluid dynamics than in the $1 \mathrm{~mm}$ and $2 \mathrm{~mm}$ Rectilinear channels. It is important to note that the viscosity, flow rate, and geometric dimensions are the same for the $1 \mathrm{~mm}$ Rectilinear and $1 \mathrm{~mm}$ Concentric Spiral channels, and yet, there is an appreciable difference in the flow properties. This suggests that difference is affected by the way in which fluid moves through each channel, for example, directly flow to the center versus the walls. Due to this impact, there should be greater delivery/transformation within the $1 \mathrm{~mm}$ Concentric Spiral channel versus the Rectilinear channels, which is supported by the Jurkat T-cell experiments discussed later.

Additionally, the average velocity was investigated at two different inlet pressures, 1 and 2 atm, with the inlet velocity held constant. Results are shown in Figure 14 and Table 9.

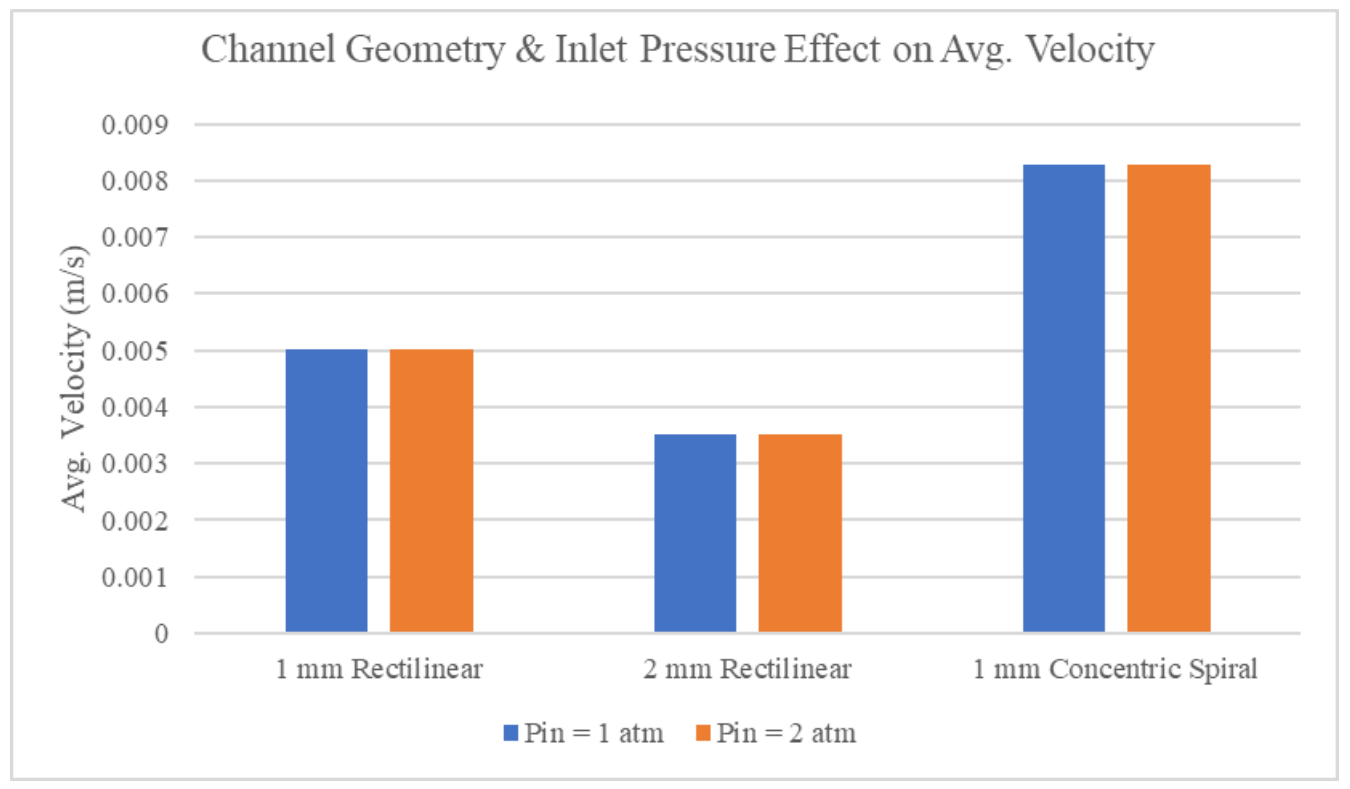

Figure 14. Comparison of volume average velocity output for each channel at both inlet pressure $=1$ and $2 \mathrm{~atm}$. Velocity follows the same trend as WSS, there is a negligible $(<1 \%)$ difference with increasing pressure, demonstrating that fluid pressure likely does not have a significant effect on the fluid dynamics.

Table 9. Volume average velocity values for each channel geometry at both Pin $=1$ and 2 atm.

\begin{tabular}{|c|c|c|}
\hline \multirow{2}{*}{ Channel } & Pin = 1 atm & Pin = 2 atm \\
\cline { 2 - 3 } & Velocity Avg. $(\mathbf{m} / \mathbf{s})$ & Velocity Avg. $(\mathbf{m} / \mathbf{s})$ \\
\hline 1 mm Rectilinear & 0.005014 & 0.005014 \\
\hline 2 mm Rectilinear & 0.003511 & 0.003511 \\
\hline 1 mm Concentric Spiral & 0.008281 & 0.008281 \\
\hline
\end{tabular}


Similar to how inlet pressure had a negligible effect on WSS, there is almost no difference in average velocity with a $1 \mathrm{~atm}$ increase in inlet pressure. Only small $(<1 \%)$ differences exist and are not detailed due to rounding of output values. Therefore, changes in the pressure will not be investigated in succeeding analyses of discrete phase particles.

The key outcome of interest in acoustofluidic sonoporation procedures is biologic delivery/cell transformation efficacy. This is affected by particle velocity/flow rate, which determines the WSS cells experience, and particle residence time, which determines the amount of US exposure. It should be noted that the particles examined in the Fluent analyses were representative of the microbubbles, not T-cells, due to the difficulty of accurately modeling T-cell properties. Contours of particle velocity were created for each channel geometry and were rescaled to a common range of $4.81 \mathrm{e}^{-04} \mathrm{~m} / \mathrm{s}$ to $4.18 \mathrm{e}^{-02} \mathrm{~m} / \mathrm{s}$. The resulting contours are shown in Figure 15 , with quantitative comparison of the minimum and maximum values highlighted in Figure 16 and Table 10.

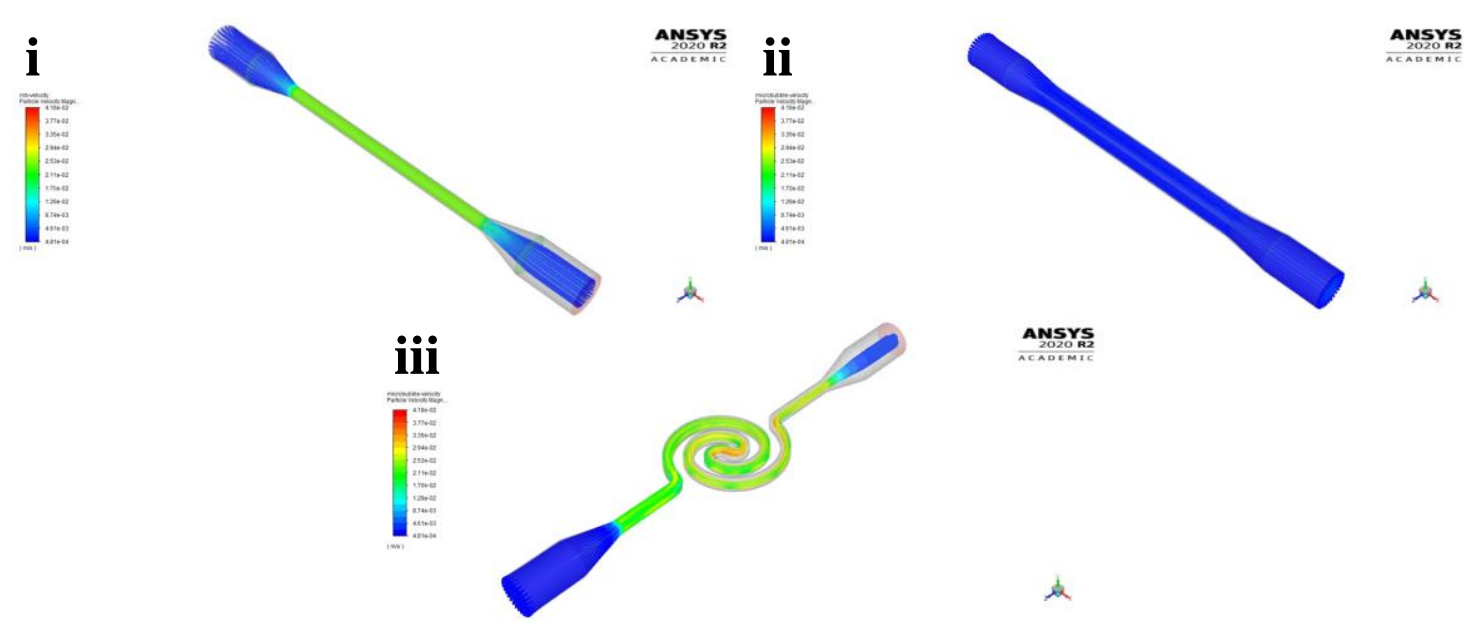

Figure 15. Scaled microbubble (MB) velocity contours for each channel geometry. The $1 \mathbf{~ m m}$ channels (Rectilinear and Concentric Spiral) generate very similar values, apart from the inner $180^{\circ}$ turn and final curve in the Concentric Spiral. This points to how both the channel size and geometry are critical factors in particle movement. 


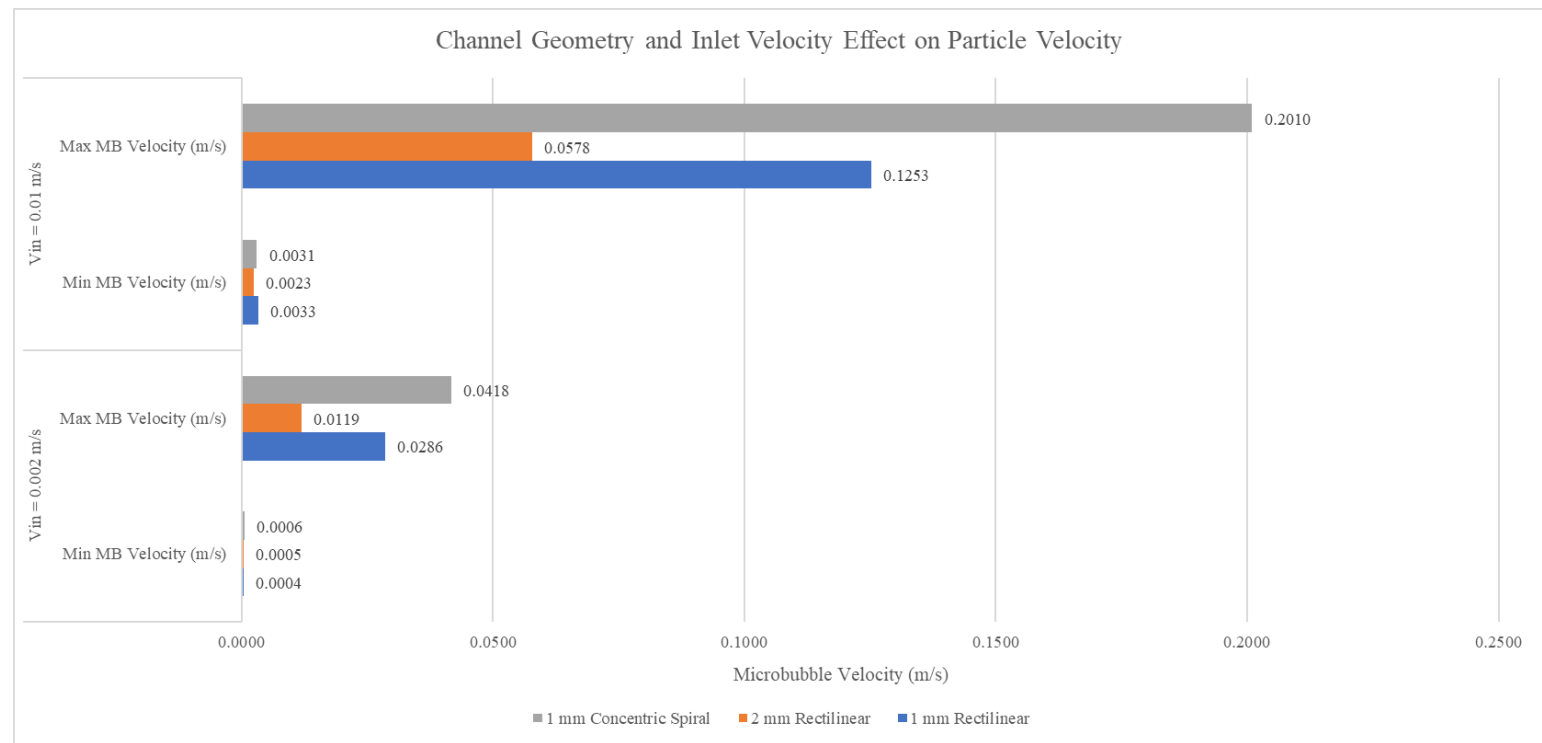

Figure 16. Comparison of minimum and maximum microbubble velocity values for each channel geometry at both Vin $=0.002$ and $0.01 \mathrm{~m} / \mathrm{s}$. The same trend detailed for both WSS and velocity is seen with particle velocity.

Table 10. Minimum and maximum microbubble velocity values for each channel and two different inlet velocities.

\begin{tabular}{|c|c|c|c|c|}
\hline \multirow{2}{*}{ Channel } & \multicolumn{2}{|c|}{ Vin = 0.002 m/s } & \multicolumn{2}{c|}{ Vin = 0.01 m/s } \\
\cline { 2 - 5 } & $\begin{array}{c}\text { Min MB Velocity } \\
(\mathbf{m} / \mathbf{s})\end{array}$ & $\begin{array}{c}\text { Max MB Velocity } \\
(\mathbf{m} / \mathbf{s})\end{array}$ & $\begin{array}{c}\text { Min MB Velocity } \\
(\mathbf{m} / \mathbf{s})\end{array}$ & $\begin{array}{c}\text { Max MB Velocity } \\
(\mathbf{m} / \mathbf{s})\end{array}$ \\
\hline $\mathbf{1 ~ m m ~ R e c t i l i n e a r ~}$ & 0.0004 & 0.0286 & 0.0033 & 0.1253 \\
\hline $\mathbf{2} \mathbf{~ m m ~ R e c t i l i n e a r ~}$ & 0.0005 & 0.0119 & 0.0023 & 0.0578 \\
\hline $\begin{array}{c}\mathbf{1} \mathbf{~ m m ~ C o n c e n t r i c ~} \\
\text { Spiral }\end{array}$ & 0.0006 & 0.0418 & 0.0031 & 0.2010 \\
\hline
\end{tabular}

In Table 10, the minimum microbubble velocity at both inlet velocities is very similar between the channels and occurs at the Inlet/Outlet_Body features, demonstrating that input conditions are similar which supports the validity of the CFD output. Like the other outputs previously considered, a $5 x$ increase in inlet velocity results in an approximately $5 x$ increase in maximum microbubble velocity. However, the trend about increasing separation between the different geometries does not entirely hold for microbubble velocity. At Vin $=0.002 \mathrm{~m} / \mathrm{s}$, the percent difference between the $1 \mathrm{~mm}$ and $2 \mathrm{~mm}$ Rectilinear channels is $140.34 \%$, but at Vin $=0.01$ 
$\mathrm{m} / \mathrm{s}$, this difference decreases to $116.78 \%$. While it is unlikely that the $2 \mathrm{~mm}$ Rectilinear channel could attain a similar value as the $1 \mathrm{~mm}$ Rectilinear channel when Vin is kept constant, this trend could be beneficial in clinical application. The $2 \mathrm{~mm}$ Rectilinear channel can likely support a greater flow rate for more time, which could result increase transformation efficiency per unit time (aka more cells transformed in the same time frame). The additional increase in flow rate could potentially create a similar particle velocity as the $1 \mathrm{~mm}$ channel geometries. While the gap in particle velocity closes between the Rectilinear channels, the previous trend of increased separation holds for the $1 \mathrm{~mm}$ Rectilinear and $1 \mathrm{~mm}$ Concentric Spiral geometries, which increases from a percent difference of $46.15 \%$ at Vin $=0.002 \mathrm{~m} / \mathrm{s}$ to $60.42 \%$ at Vin $=0.01 \mathrm{~m} / \mathrm{s}$. As seen in Figure 14.iii, the peak particle velocity occurs at the center $180^{\circ}$ turn, with increased values occurring again at the last curve just prior to the outlet. Other interesting phenomena that can be seen in these results is the spread of particles at the outlet of each channel. In the $1 \mathrm{~mm}$ Rectilinear channel, particles nearly fill the volume of the Outlet_Body feature. In the $2 \mathrm{~mm}$ Rectilinear channel, particles fill the entire volume, likely due to the limited decrease in channel diameter/height between the inlet and channel. However, in the $1 \mathrm{~mm}$ Concentric Spiral geometry, the particles stay fairly condensed in the center of the outlet. While it is unlikely these flow patterns influence cell transformation/biologic delivery, this information could be beneficial in designing channels to direct particles in a specific direction. Likely, particle density could influence the movement, which could be adapted to serve as a sorting mechanism for collecting transformed cells, untransformed cells, and unruptured microbubbles, assuming a large enough variation in density. The spread of particles at the outlet of each channel are shown in Figure 17. 

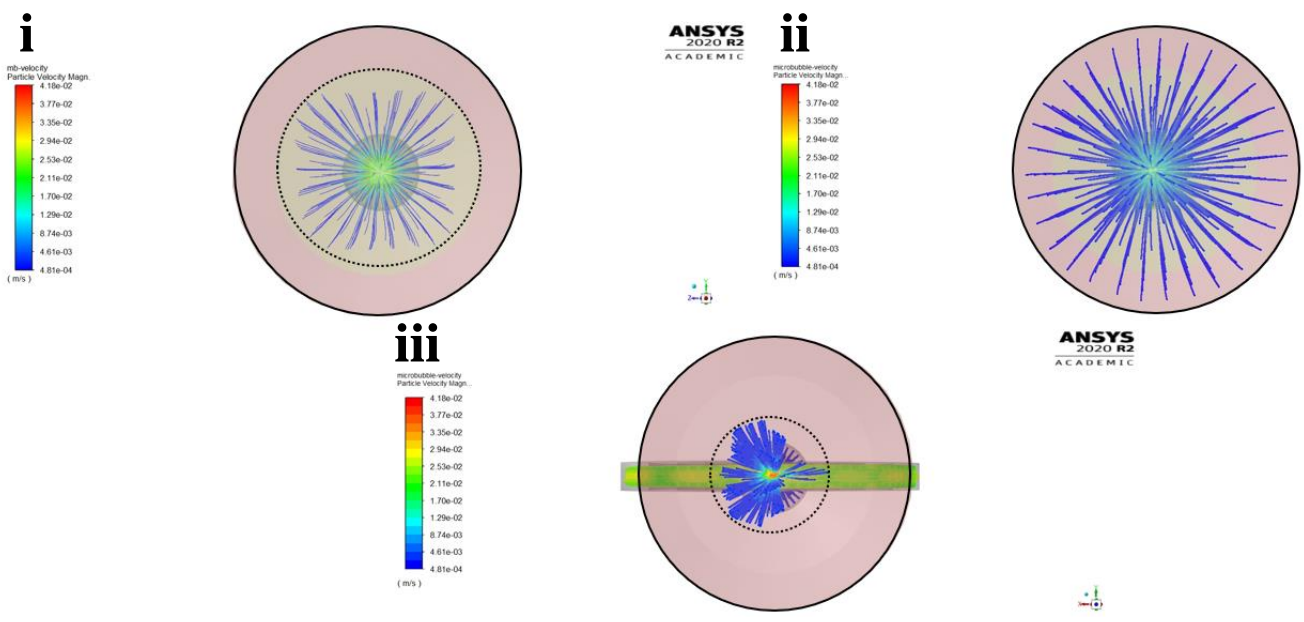

Figure 17. Images of the particle distribution at the outlet of each channel. The solid black line denotes the outer wall of the outlet while the dashed black line denotes the area in which particles are contained. Even though velocity values are similar for each channel, a unique spread is generated.

i) $1 \mathrm{~mm}$ Rectilinear ii) $2 \mathrm{~mm}$ Rectilinear iii) $1 \mathrm{~mm}$ Concentric Spiral

Particle residence time was also compared between the channels at Vin $=0.002 \mathrm{~m} / \mathrm{s}$, with a common scale of $0 \mathrm{~s}$ to $5.09 \mathrm{e}^{01} \mathrm{~s}$. Contours of particle residence time are shown in Figure 18 while quantitative comparisons are presented in Figure 19 with histograms of the distribution of residence time and Table 11 which contains general statistics (min, max, mean, and standard deviation) for each distribution.

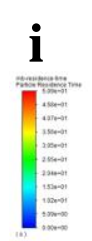

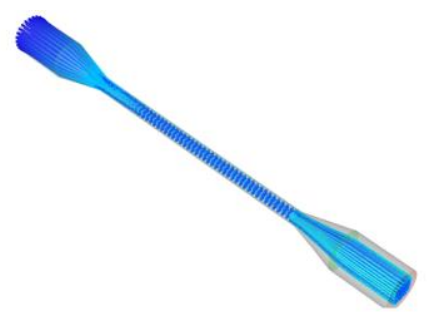

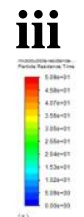

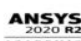
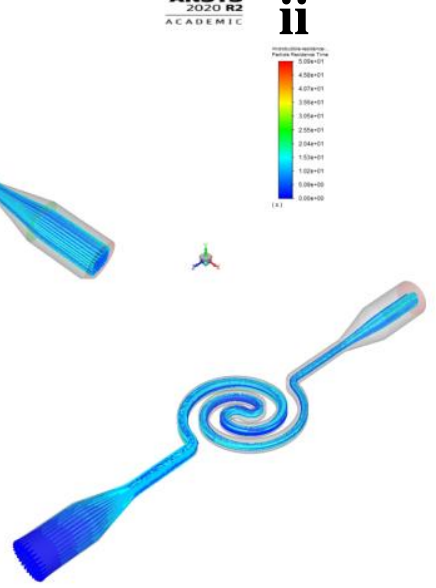

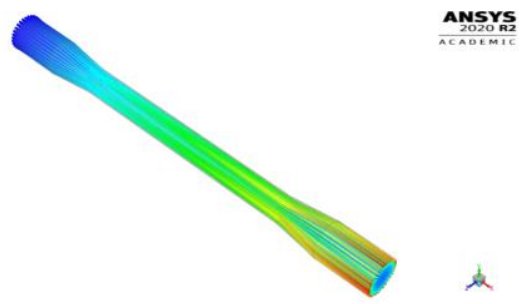

ANSYS

SYS 

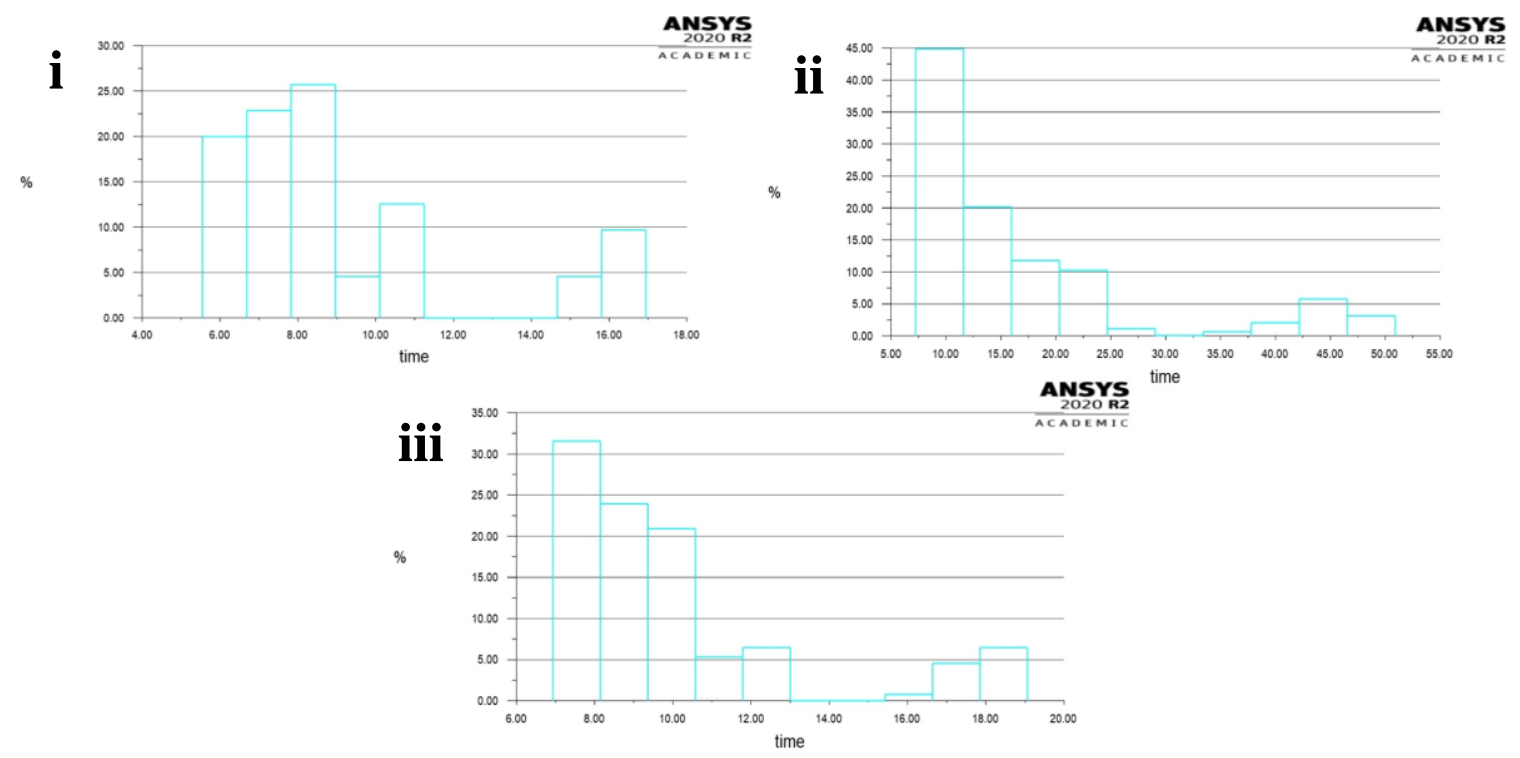

Figure 19. Histograms of particle residence time for each channel. These reveal a slight bimodal distribution for each channel; the majority of particles exit quickly, however, anywhere between 10$15 \%$ exit in significantly longer times. It is important to note that these potential outliers could skew metrics such as the mean.

Table 11. Key metrics for each channel's microbubble residence time histogram.

\begin{tabular}{|c|c|c|c|}
\hline & \multicolumn{3}{|c|}{ Residence Time (s) } \\
\hline & $\mathbf{1 ~ m m}$ Rectilinear & $\mathbf{2 ~} \mathbf{~ m m}$ Rectilinear & $\mathbf{1 ~ m m ~ C o n c e n t r i c ~ S p i r a l ~}$ \\
\hline Mean & 9.08999 & 16.6448 & 10.0313 \\
\hline Standard Deviation & 3.16481 & 11.1707 & 3.17839 \\
\hline Min & 5.54721 & 7.2262 & 6.93633 \\
\hline Max & 16.9441 & 50.8997 & 19.0634 \\
\hline
\end{tabular}

The particle residence time results reveal that the $1 \mathrm{~mm}$ Rectilinear and $1 \mathrm{~mm}$ Concentric Spiral channels share a very similar residence time spread, despite having vastly different particle velocities. There is only a $10.36 \%$ difference in the mean residence time at Vin $=0.002 \mathrm{~m} / \mathrm{s}$, yet a 46.15\% difference in max velocity. This has important considerations as the $1 \mathrm{~mm}$ Concentric Spiral channel is longer than the $1 \mathrm{~mm}$ Rectilinear channel, yet particles move through each in the same time. This implies that particles spend a greater amount of time within the US field, due to increased overlap with the ultrasound transducer, and experience greater shear stress in the $1 \mathrm{~mm}$ Concentric Spiral channel, which should result in greater biologic delivery. 
Additionally, the core of each channel has the lowest residence time, which is comparable between all the channel geometries; the differences arise when particles begin to spread to the outer edge of the outlet. While there is a maximum of $50.9 \mathrm{~s}$, with $\sim 10 \%$ of particles having a residence time $>30 \mathrm{~s}$, based on the histogram in Figure 18.ii, $45 \%$ of the particles in the $2 \mathrm{~mm}$ Rectilinear channel exit in $<12.50 \mathrm{~s}$. This variation is explained by analyzing the microbubble velocity distribution at the outlet in unscaled images of the different geometries, as shown in Figure 20. Compared to the $1 \mathrm{~mm}$ channel geometries, there is a velocity difference between the core/center of the flow and outer edge in the $2 \mathrm{~mm}$ Rectilinear channel. Using relative values for the velocity, the center is approximately $3.90 \mathrm{e}^{-03} \mathrm{~m} / \mathrm{s}$ versus the outer edge at $4.81 \mathrm{e}^{-04} \mathrm{~m} / \mathrm{s}$, a $710.81 \%$ difference. This significant difference results in a wider range for particle residence time, as compared to the other geometries.
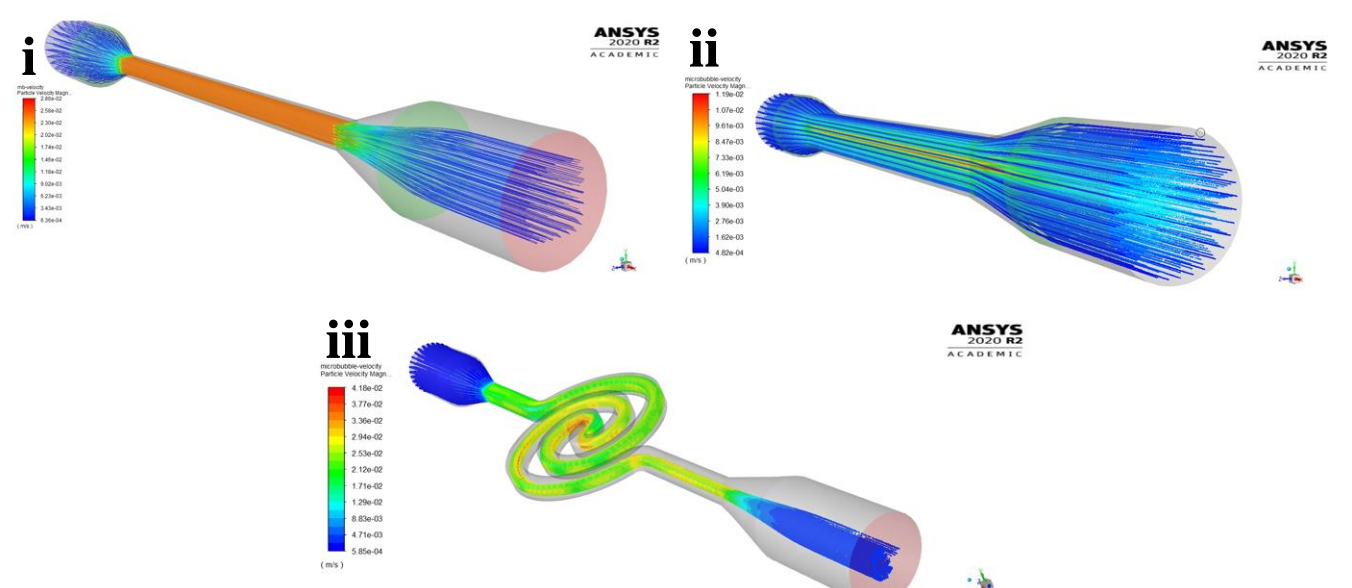

Figure 20. Unscaled images of microbubble velocity, focused on the outlet, for each channel. In both $1 \mathrm{~mm}$ configurations, there is little to no difference in the particle velocity throughout the outlet. However, in the $2 \mathbf{~ m m}$ Rectilinear channel, a distinct difference can be seen from the center to the outer edge. 


\section{Jurkat T-Cell Transfection Experiments}

Results of the first set of Jurkat T-cell biologic delivery experiments are shown in Figure 21 and Table 12 and 13.
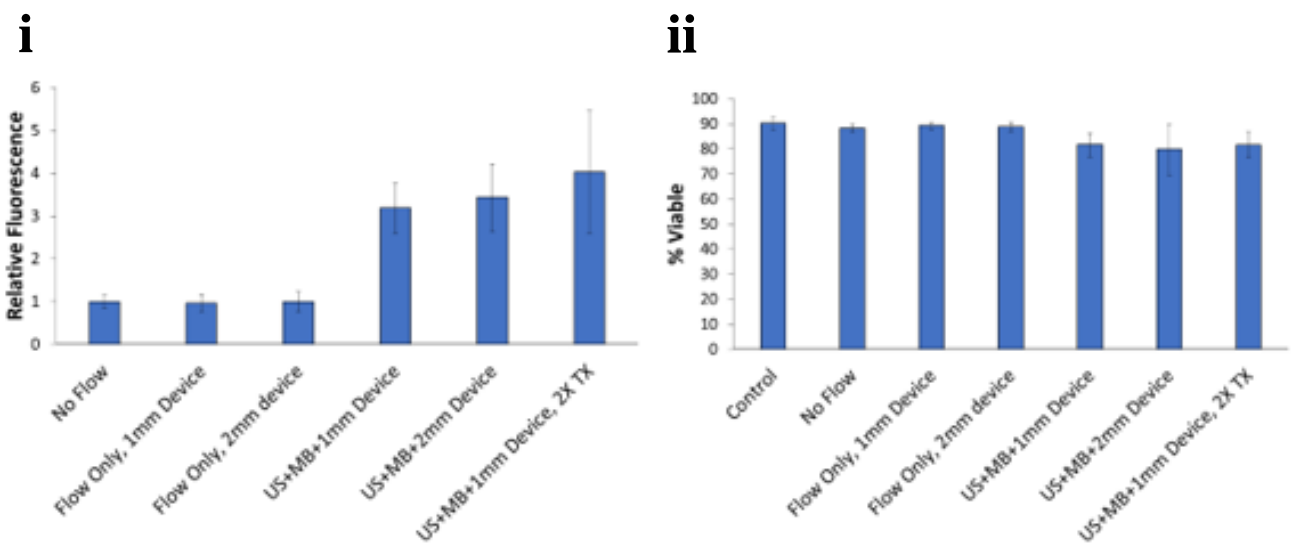

Figure 21. i) Relative calcein fluorescence between the difference experimental groups $(n=3)$ for the first set of transformation experiments. An ANOVA analysis revealed that US is the only significant

factor in altering calcein delivery $(p<0.001)$ ii) Percent viability $(1-$ PI intensity) for each experimental group. An ANOVA analysis revealed that the inclusion of US significantly reduced viability $(p<0.001)$, however, all viabilities remained $>75 \%$.

Table 12. Relative (to No Flow condition) calcein fluorescence mean and standard deviation for the six experimental groups.

\begin{tabular}{|c|c|c|c|c|c|c|}
\hline & & \multicolumn{2}{|c|}{ Flow Only } & \multicolumn{3}{c|}{ US Treatment } \\
\hline & $\begin{array}{c}\text { No } \\
\text { Flow }\end{array}$ & $\begin{array}{c}\text { 1mm } \\
\text { Device }\end{array}$ & $\begin{array}{c}\mathbf{2 m m} \\
\text { device }\end{array}$ & $\begin{array}{c}\text { 1mm } \\
\text { Device }\end{array}$ & $\begin{array}{c}\mathbf{2 m m} \\
\text { Device }\end{array}$ & $\begin{array}{c}\text { 1mm Device, } \\
\text { 2X TX }\end{array}$ \\
\hline $\begin{array}{c}\text { Average Calcein } \\
\text { Fluorescence (xNo Flow) }\end{array}$ & 1.0000 & 0.9612 & 0.9947 & 3.1873 & 3.4340 & 4.0250 \\
\hline $\begin{array}{c}\text { Standard Deviation of } \\
\text { Calcein Fluorescence }\end{array}$ & 0.1575 & 0.2024 & 0.2307 & 0.5888 & 0.7900 & 1.4355 \\
\hline
\end{tabular}

Table 13. Cell viability mean and standard deviation for the seven experimental groups.

\begin{tabular}{|c|l|l|c|c|c|c|c|}
\hline & & & \multicolumn{2}{|c|}{ Flow Only } & \multicolumn{3}{c|}{ US Treatment } \\
\hline & Control & No Flow & $\begin{array}{c}\mathbf{1 m m} \\
\text { Device }\end{array}$ & $\begin{array}{c}\mathbf{2 m m} \\
\text { Device }\end{array}$ & $\begin{array}{c}\mathbf{1 m m} \\
\text { Device }\end{array}$ & $\begin{array}{c}\mathbf{2 m m} \\
\text { Device }\end{array}$ & $\begin{array}{c}\mathbf{1 m m} \text { Device, } \\
\text { 2X TX }\end{array}$ \\
\hline $\begin{array}{c}\text { Average Cell } \\
\text { Viability (\%) }\end{array}$ & 90.1600 & 88.2767 & 89.2750 & 88.8517 & 81.6333 & 79.8500 & 81.5167 \\
\hline $\begin{array}{c}\text { Standard } \\
\text { Deviation of } \\
\text { Cell Viability }\end{array}$ & 2.3857 & 1.6285 & 1.4299 & 1.8306 & 4.7807 & 10.1903 & 5.1211 \\
\hline
\end{tabular}


Analysis of the relative calcein fluorescence in Figure 21.i highlights that while there may be a nearly 10x increase in volume average WSS (0.01151 Pa vs. $0.00110 \mathrm{~Pa})$ between the $1 \mathrm{~mm}$ and $2 \mathrm{~mm}$ Rectilinear channels, there is no significant difference in calcein delivery. Even with the addition of US, whose effect should be relative to the residence time, there is no significant difference. The seventh group (US+MB+1mm Device, $2 \mathrm{X}$ TX) was used to address for the nearly $2 \mathrm{x}$ difference in mean particle residence time between the channels and still did not result in a significant change in delivery. These results suggest that WSS has a negligible effect on delivery efficacy in these acoustofluidic channels, the main determinant is the inclusion of US. While literature has shown that flow alone in microfluidic channels can result in significant cell transformation ${ }^{[23,24]}$, in the larger fluidic channels, the WSS can likely not reach the necessary threshold to permeabilize cells.

The second biologic delivery experiments were performed to compare calcein delivery and cell viability between all three channel geometries. Graphical comparisons of relative calcein fluorescence and cell viability are shown in Figure 22, with the values given in Table 14 and 15.
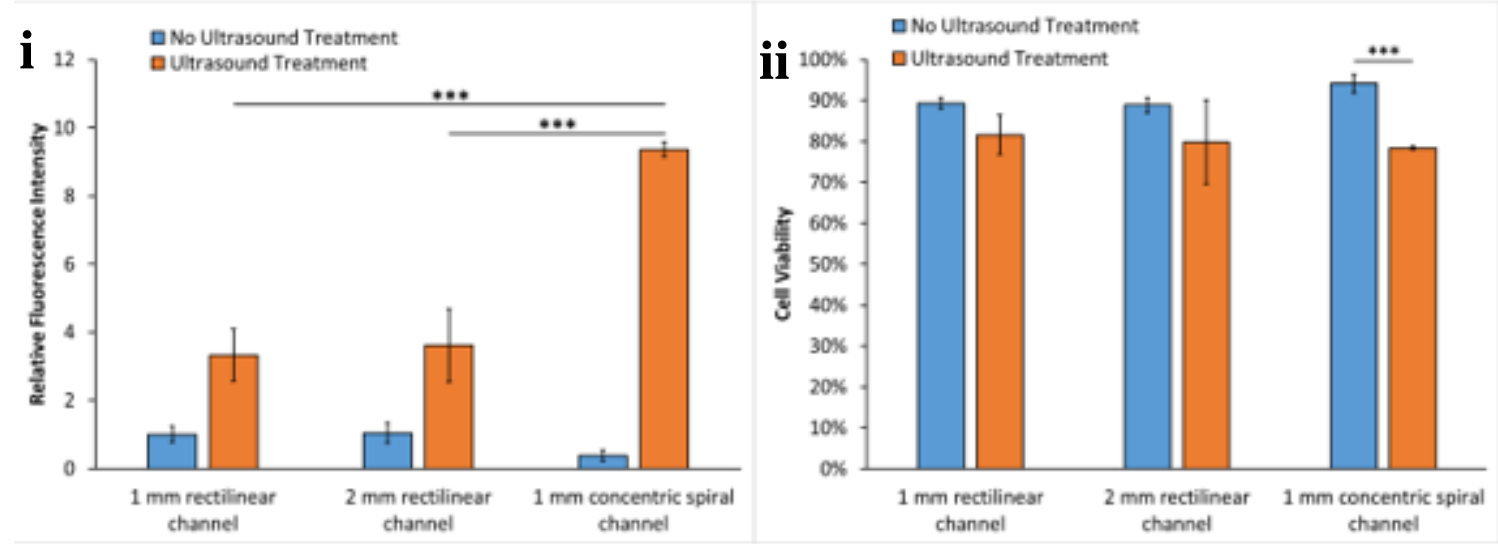

Figure 22. i) Comparison of the channel geometries with and without ultrasound treatment $(n=$ 6/group). Ultrasound treatment significantly enhanced calcein delivery to Jurkat $T$-cells in each device $(p<0.001)$. Ultrasound treatment enhanced calcein delivery to Jurkat T-cells in the $1 \mathbf{~ m m}$ Concentric Spiral channel compared to both the $1 \mathrm{~mm}$ and $2 \mathrm{~mm}$ Rectilinear channels $(p<0.001)$. ii) Ultrasound treatment reduced cell viability $(1-$ PI intensity) in fluidic devices $(p<0.001)$, however, all groups remained above $75 \%$. 
Table 14. Relative (to $1 \mathrm{~mm}$ Rectilinear, Flow Only) calcein fluorescence mean and standard deviation for the six experimental groups.

\begin{tabular}{|c|c|c|c|c|c|c|}
\hline & \multicolumn{3}{|c|}{ Flow Only } & \multicolumn{3}{c|}{ US Treatment } \\
\hline & $\begin{array}{c}\mathbf{1 ~ m m} \\
\text { Rect. }\end{array}$ & $\begin{array}{c}\mathbf{2 ~ m m} \\
\text { Rect. }\end{array}$ & $\begin{array}{c}\mathbf{1 ~ m m} \\
\text { Spiral }\end{array}$ & $\begin{array}{c}\mathbf{1 ~ m m} \\
\text { Rect. }\end{array}$ & $\begin{array}{c}\mathbf{2} \mathbf{~ m m} \\
\text { Rect. }\end{array}$ & $\begin{array}{c}\mathbf{1 ~ m m} \\
\text { Spiral }\end{array}$ \\
\hline $\begin{array}{c}\text { Average Calcein Fluorescence } \\
\text { (x1mm Rectilinear Flow Only) }\end{array}$ & 1.0000 & 1.0443 & 0.3781 & 3.3321 & 3.6111 & 9.3617 \\
\hline $\begin{array}{c}\text { Standard Deviation of Calcein } \\
\text { Fluorescence }\end{array}$ & 0.2394 & 0.3018 & 0.1656 & 0.7605 & 1.0730 & 0.2056 \\
\hline
\end{tabular}

Table 15. Cell viability mean and standard deviation for the six experimental groups.

\begin{tabular}{|c|c|c|c|c|c|c|}
\hline & \multicolumn{3}{|c|}{ Flow Only } & \multicolumn{3}{c|}{ US Treatment } \\
\hline & $\begin{array}{c}\mathbf{1 ~ m m} \\
\text { Rect. }\end{array}$ & $\begin{array}{c}\mathbf{2} \mathbf{~ m m} \\
\text { Rect. }\end{array}$ & $\begin{array}{c}\mathbf{1 ~ m m} \\
\text { Spiral }\end{array}$ & $\begin{array}{c}\mathbf{1 ~ m m} \\
\text { Rect. }\end{array}$ & $\begin{array}{c}\mathbf{2} \mathbf{~ m m} \\
\text { Rect. }\end{array}$ & $\begin{array}{c}\mathbf{1 ~ m m} \\
\text { Spiral }\end{array}$ \\
\hline Average Cell Viability (\%) & 89.28 & 88.85 & 94.14 & 81.63 & 79.85 & 78.43 \\
\hline $\begin{array}{c}\text { Standard Deviation of Cell } \\
\text { Viability }\end{array}$ & 1.43 & 1.83 & 2.25 & 4.78 & 10.19 & 0.45 \\
\hline
\end{tabular}

Figure 22.i supports the results demonstrated in the first set of cell experiments, flow alone does not produce a significant increase in calcein delivery and even large differences in WSS between the different geometries does not create appreciable differences. An interesting aspect to note about these results is that the $1 \mathrm{~mm}$ Concentric Spiral channel had a reduced relative fluorescence compared to both Rectilinear channels in the flow only condition, despite having the greatest volume average WSS; this trend is reversed however once US is added, likely due to the increased US exposure. Additionally, the conclusion that US is the main determinant in delivery is supported be the figure, as the inclusion of US increases the relative fluorescence by at least 3-fold in all the channels. The significant $(p<0.001)$ difference between the Rectilinear channels and the Concentric Spiral channel is likely driven by the increased US exposure due to the increased length of the Concentric Spiral channel. As discussed previously, while residence time is comparable between both $1 \mathrm{~mm}$ configurations, the increased length and increased overlap of the ultrasound transducer in the Concentric Spiral geometry creates a greater opportunity for microbubble rupture and cell permeabilization. It should be noted that even with a 9x increase in relative fluorescence, cell viability remained $>75 \%$ in the Concentric Spiral geometry. 
To explore potential connections between the CFD simulation results and cell experiment results from experiment 2, Figure 23 displays the percent difference in average WSS and relative calcein fluorescence between the $1 \mathrm{~mm}$ and $2 \mathrm{~mm}$ Rectilinear channels (left) and between the 1 mm Rectilinear and $1 \mathrm{~mm}$ Concentric Spiral channels (right).

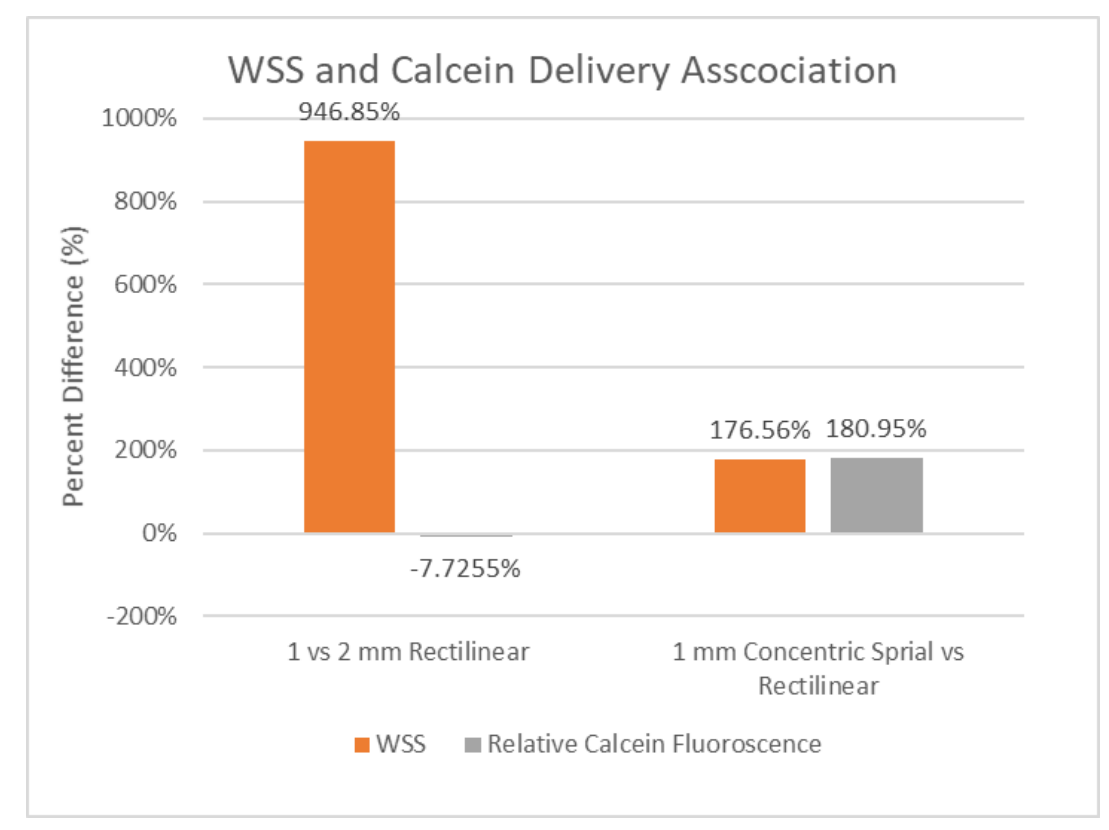

Figure 23. Comparison of average WSS and Relative Calcein Fluorescence between the channel geometries. While a 946\% difference exists between the 1- and 2-mm Rectilinear channels, there is a slightly decreased fluorescence in the $1 \mathrm{~mm}$ diameter channel compared to the $\mathbf{2} \mathbf{~ m m}$ diameter channel. However, there is a smaller difference in average WSS between the $1 \mathrm{~mm}$ Rectilinear and Concentric Spiral yet there is a significant increase in calcein fluorescence, contrary to the trend noted with the Rectilinear geometries. These results suggest that average WSS may not be the main determinant in biologic delivery.

As noted previously, while there is a stark difference in the average WSS and velocity magnitude between the Rectilinear geometries, there is no significant difference in calcein delivery, likely due to minimal overlap with the US transducer. Particles may spend up to $17 \mathrm{~s}$ or $51 \mathrm{~s}$ in the $1 \mathrm{~mm}$ and $2 \mathrm{~mm}$ Rectilinear channels, respectively, but much of this time is in the inlet and outlet where no US is applied. On the other hand, the differences in average WSS is smaller between the $1 \mathrm{~mm}$ Rectilinear and Concentric Spiral geometries; but there is a significant difference in calcein fluorescence likely driven by the increased path length and overlap with the US transducer. It 
should be noted that these conclusions are complicated by the fact the CFD simulations assumed steady flow (such as from a syringe pump) while the cell experiments were conducted using a peristaltic pump; therefore, pulsatile flow could affect these results and complicate the comparison of each analysis. Despite this limitation, these results suggest that average WSS may not be the primary factor in acoustofluidic-mediated molecular delivery. 


\section{CONCLUSIONS}

Comparing both the CFD and cell experiment results highlights that the $1 \mathrm{~mm}$ Concentric Spiral channel is currently the optimal channel design for maximizing biologic delivery. However, this is likely caused by greater overlap of the US transducer with the channel yielding increased US exposure compared to either Rectilinear geometry. Based on the results shown in Figures 21.i and 22.i, US is the main determinant in altering biological delivery within the fluidic channels. To be more specific, it appears that the $180^{\circ}$ turn is the geometry feature that sets the $1 \mathrm{~mm}$ Concentric Spiral apart from the $1 \mathrm{~mm}$ Rectilinear channel. While difficult to see in Figure 7 , the $180^{\circ}$ turn produces the peak volume average WSS value and as shown in Figure 15, similar particle velocities are achieved throughout both the $1 \mathrm{~mm}$ Rectilinear and $1 \mathrm{~mm}$ Concentric Spiral channels except at this center turn, and even more revealing, this significant spike in particle velocity does not decrease particle residence time. These findings suggest that a series of parallel $180^{\circ}$ turns could be an effective channel design for increasing the WSS, residence time, and US exposure; an example of this proposed channel is shown in Figure 24.

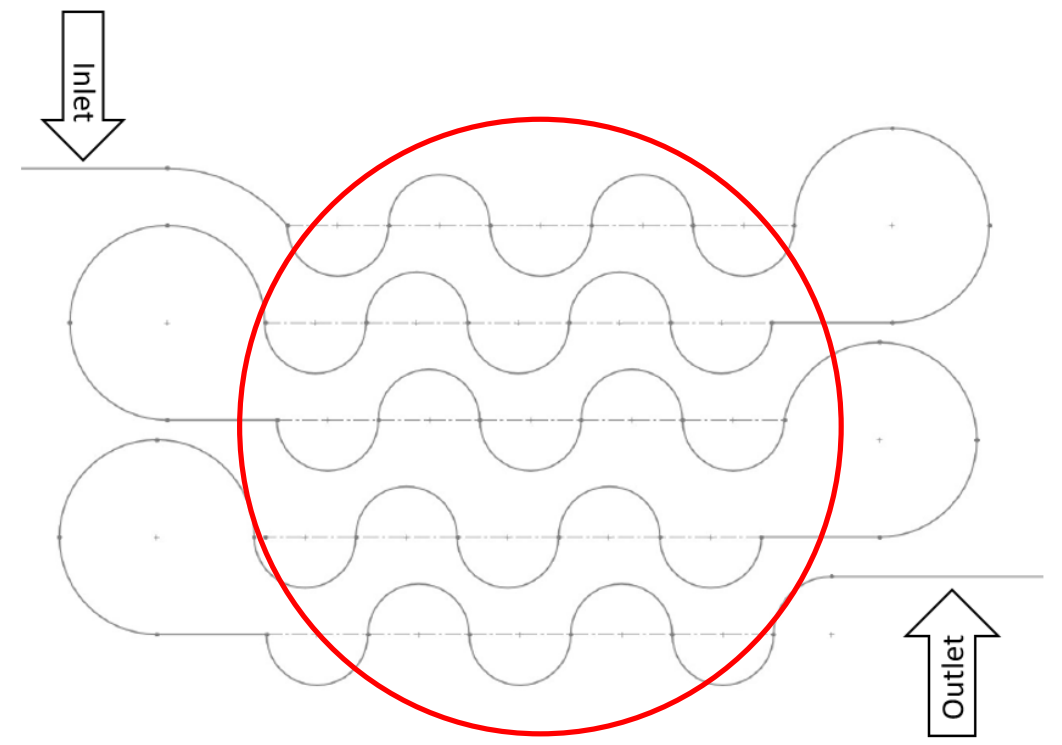

Figure 24. Potential design for channel with multiple $180^{\circ}$ turns. The red circle denotes how a circular US transducer could be utilized to cover much of the volume, depending on size. 
Aside from suggesting an optimal geometry, these results demonstrate two other important conclusions: 1) inlet pressure has a negligible effect on the fluid dynamics and 2) a flow rate of 1.5 $\mathrm{mL} / \mathrm{min}(\mathrm{Vin}=0.002 \mathrm{~m} / \mathrm{s})$ is not great enough to generate WSS values that significantly alters membrane permeability without US exposure. Figures 9 and 14 highlight that fluid pressure does not significantly alter the fluid dynamics as the output values only vary by at most $1 \%$ when increasing Pin from 1 to $2 \mathrm{~atm}$. While not unexpected knowing that the defining equation for WSS in a laminar flow regime does not include a variable for pressure, there were no changes to particle flow position/pattern with changes in pressure. Figures 21.i and 22.i point to how the WSS can likely not reach some threshold value to significantly alter membrane permeability within these larger, millimeter sized channels. However, WSS may be important in sensitizing cells to the acoustofluidic treatment, which could explain the 9-fold increase in calcein fluorescence seen in Figure 22.i. Since all the values in Equation 1 are constant, except for flow rate, $Q$, only increases in flow rate could potentially shift WSS into a range that would result in significant biologic delivery.

There are a number of limitations and refinements to the present analyses that should be addressed in future CFD modeling. In this analysis, the entire channel volume was utilized to calculate the output parameters, specific features, regions, sections, etc. could not be investigated and compared individually due to the channels being modeled in SOLIDWORKS and imported into ANSYS. To address this limitation, the channels should be designed within ANSYS DesignModeler so more specificity could be applied in analysis. Seen throughout the analyses presented, the main differences present within the Channel feature, however calculated values include the inlet/outlet portions. If the channels were built within DesignModeler, the values within the Channel could be extracted independently, removing the inlet/outlet artifact, thereby producing more accurate results.

Another limitation in the current work was the inclusion of only one discrete phase model, when at least two were present in the biologic delivery experiments. While the microbubble 
injection is likely representative of the actual microbubbles used, they are likely not representative of the Jurkat T-cells which have a larger diameter $(5-7 \mu \mathrm{m})$, a different density, and may not follow all the same laws/dynamics. Therefore, the flow patterns seen likely do not describe Jurkat T-cell dynamics accurately. Additionally, the interactions between microbubbles and Jurkat T-cells could not be captured in the present simulations. Collisions could represent a significant factor in biologic delivery efficiency, as the closer a microbubble is to a cell when it ruptures exponentially increases the shear the cell experiences. If there is a different flow pattern for each particle, finding geometries that favor mixing/interaction could be the next step in further increasing biologic delivery consistency and efficacy.

Finally, the assumption of a laminar flow regime within the fluidic channels may not be applicable. The actual flow regime should be explored through high-speed photography to determine what type is present. ANSYS Fluent offers a wide range of turbulence models (ex. Spalart-Allmares, $\mathrm{k}-\varepsilon, \mathrm{k}-\omega$, etc.), so it is likely that a model will fit with the actual flow regime. If flow is found to not be laminar, the current results are essentially invalid, but do provide a useful background. It should be relatively simple to change the Viscous model within each simulation and re-do the analyses, thereby producing more valid results.

Future work should center around investigating the WSS threshold value that permeabilizes cells and produces a significant increase in biologic delivery without US. Alongside calculating the value, the duration in which a cell needs to be exposed to this WSS should also be investigated. After determining these values, the trends presented in Figure 10 could be utilized to calculate the necessary inlet flow rate/velocity that will create the necessary WSS which could then be coupled with a channel geometry that exposes them for the necessary duration (e.g. a narrow, short tube to maximize WSS for enhanced permeabilization of cells followed by a larger geometry to slow particles and increase residence time within the US beam). Currently, it appears that flow is not necessary for transformation, and applying US to a static cell suspension in a vial could potentially produce similar trends; essentially, flow does not appear to be a significant parameter for molecular 
delivery with this approach. However, making use of flow to supplement the US effect would increase throughput, biologic delivery consistency, and potentially support acoustofluidic sonoporation as becoming a gold-standard for cell transformation and assist its clinical translation. Additionally, knowing the needed WSS would aid in channel design optimization.

Two other refinements to the current models include investigating transient/pulsatile flow, as driven by a peristaltic pump, versus steady flow, as driven by a syringe pump. Pulsatile flow could alter flow rate creating regions of increased/decreased velocity that would change WSS and US exposure and drive discrete particles into specific flow patterns to favor interactions/collisions. If changes in flow rate were significant, pulsatile flow could move the entire flow regime from laminar to turbulent and vice-versa, which has the potential to create unique flow patterns of swirling/mixing. Combined with changing geometry (ex. a smaller followed by a larger diameter), this presents the best optimization scheme. Finally, simulating the US effects would greatly improve the correlation ability of the CFD calculations to the cell experiment results. ANSYS Fluent offers the ability to model acoustics which could address this gap; however, a much more in-depth knowledge of the necessary modeling and setup is needed to incorporate acoustics. As noted previously, research has shown that acoustic radiation force effects generated by US application can alter the position in which particles flow through the channel, which would likely change the DPM Concentration contours presented in Figure 11. This movement likely influences the flow dynamics and shear stresses that particles experience, especially since this movement is typically towards the opposite wall of the transducer which should also increase the proportion of particles that experience a significant amount of WSS.

Taken together, these conclusions highlight that this thesis only began to scratch the surface of understanding the fluidic forces within acoustofluidic channels. Geometries that alter the direction of flow (e.g. $180^{\circ}$ turn) versus straight channels appear to be effective in increasing the WSS particles experience, cause a large increase in particle velocity without significantly changing residence time, and therefore, result in higher biologic delivery. US exposure currently appears to 
be the most significant factor in improving biologic delivery within 1- and 2-mm channels, therefore, channel geometry should be tailored to maximize residence time. Future research should focus on validity the flow regime in acoustofluidic channels to ensure accuracy of CFD generated results and adding additional parameters to the CFD models (e.g. multiple discrete phase models). Additional refinement of these models could reveal new details and trends that will be important for translating this technology into clinical practice for cell transformation. 


\section{RECOMMENDATIONS}

Acoustofluidic sonoporation is a promising alternative for cell transformation, however, there is still a significant amount of work needed to fully understand the physical forces and fluid dynamics that drive cell permeabilization in the devices. The work presented in this thesis details a beginning foundation for which to direct future analyses and experimentation. These analyses should be repeated with additional computing power and a non-academic version of ANSYS to test mesh element sizes smaller than $0.2 \mathrm{~mm}$. While the mesh independence analyses began to reach steady-state values for the various output parameters, further refinement should provide more accurate results that will result in a more thorough understanding of the fluid dynamics. Additionally, with more computing power, the Jurkat-T-cells and microparticles could be added as discrete phase models and interactions, whether DEM or stochastic collisions, could be introduced. This information could reveal unique flow patterns for each type of particle; how interactions affect velocity, residence time, and other variables; and better correlate CFD calculations to cell experiment results. For example, new research is suggesting that the use of cationic microbubbles versus neutral microbubbles, results in a "stickiness" to Jurkat T-cells that increases permeability and biologic delivery ${ }^{[18]}$. Building Jurkat T-cells into the ANSYS Fluent analyses and adding a "sticky" collision with the microbubbles has the potential to significantly improve acoustofluidic sonoporation knowledge. This information would be immensely useful in designing fluidic channels that drive particles to interact, maximizing WSS and US exposure, while increasing throughput.

As analyses are refined, more biologic delivery experiments should be conducted. Variables other than channel geometry should be investigated such as inlet flow rate/velocity, inlet pressure, US exposure time, US pressure, and more. Based on both the CFD output and cell experiments, the shear stress generated by flow alone is not enough to permeabilize cells effectively; it is the inclusion of US that significantly improves delivery. Greater flow rates should 
be tested to understand if there is a threshold that results in a significant change in biologic delivery, which could then be modeled in ANSYS to develop a WSS threshold. If there are significant differences in delivery with changes to inlet pressure, the results of this analysis would no longer be valid as a laminar flow regime does not accurately capture this effect. This information would be extremely beneficial for refining the CFD models. Eventually, with significant agreement between the CFD models and cell experiment results, the models could be used for predicting delivery and viability results in new channel geometries prior to them being printed and tested.

Moving forward, to maximize transformation, the $1 \mathrm{~mm}$ Concentric Spiral with US should be used. Pooling all the results presented suggests that out of the three geometries, it is the optimal design currently. The cell suspension should be pumped at the maximum flow rate/velocity possible, adjusting as necessary to maintain a cell viability $\geq 75 \%$. If results demonstrate a significant decrease in biologic delivery, this is likely caused by a decrease in residence time that limits US exposure; multiple channel flow throughs (ex. 2X, 3X) could be used to address this decrease. To refine the $1 \mathrm{~mm}$ Concentric Spiral as it currently stands, the channel should be made smaller (ex. $200 \times 200 \mu \mathrm{m}$ ) and the number of turns (especially $180^{\circ}$ ) should be increased. These two alterations should both increase the WSS cells experience, by changing the geometric quantities $W$ and $h$ in Equation 1, and US exposure time, by increasing the length of the channel. The potential for decreasing the size of the channel is currently limited by current SLA 3D printing techniques, but it is very feasible to add additional spirals.

Finally, the potential for sorting transformed cells, untransformed cells, and unruptured microbubbles by altering final curve and outlet geometry should be investigated. This could be accomplished by adding multiple smaller outlets spaced in some specific spatial orientation (ex. horizontally) that would capture the flow of particles of a certain density. Untransformed cells and unruptured microbubbles could be looped back into circulation to reduce the need for postprocessing separatory techniques and increase overall transformation efficacy. Especially if the flow regime is not laminar, the swirling/mixing that occurs in turbulent flow regimes could be used 
to aid in the separation process. Like column chromatography, pulsatile flow at varying velocities could force different particles out of the channel at different times.

Therefore, for present operations/experiments, the $1 \mathrm{~mm}$ Concentric Spiral should be used at greater flow rates with additional flow throughs; if possible, the geometry should be SLA printed at smaller diameters. These small alterations in the current procedures/setup should significantly increase the WSS and microbubble rupture stress that cells experience, which should continue to increase biologic delivery. It is important to keep in mind however, that too much shear exposure could adversely affect delivery results, so small, incremental changes in these parameters should be tested. While there are still improvements that can be made, based on the work presented here, this scheme is the current optimal configuration. 


\section{REFERENCES}

[1] U.S. Food and Drug Administration. (2017, August 30). FDA approval brings first gene therapy to the United States [Press Release]. https://www.fda.gov/news-events/press-announcements/fdaapproval-brings-first-gene-therapy-united-states

[2] U.S. Food and Drug Administration. (2017, October 18). FDA approves CAR-T cell therapy to treat adults with certain types of large B-cell lymphoma [Press Release]. https://www.fda.gov/newsevents/press-announcements/fda-approves-car-t-cell-therapy-treat-adults-certain-types-large-bcell-lymphoma

[3] U.S. Food and Drug Administration. (2020, July 24). FDA Approves First Cell-Based Gene Therapy For Adult Patients with Relapsed or Refractory MCL [Press Release]. https://www.fda.gov/newsevents/press-announcements/fda-approves-first-cell-based-gene-therapy-adult-patients-relapsedor-refractory-mcl

[4] Abbaci, M., Barberi-Heyob, M., Blondel, W., Guillemin, F., \& Didelon, J. (2008). Advantages and limitations of commonly used methods to assay the molecular permeability of gap junctional intercellular communication. Biotechniques, 45(1), 33-52, 56-62.

https://doi.org/10.2144/000112810

[5] Belling, J. N., Heidenreich, L. K., Tian, Z., Mendoza, A. M., Chiou, T. T., Gong, Y., Chen, N. Y., Young, T. D., Wattanatorn, N., Park, J. H., Scarabelli, L., Chiang, N., Takahashi, J., Young, S. G., Stieg, A. Z., De Oliveira, S., Huang, T. J., Weiss, P. S., \& Jonas, S. J. (2020). Acoustofluidic sonoporation for gene delivery to human hematopoietic stem and progenitor cells. Proc Natl Acad Sci U S A, 117(20), 10976-10982. https://doi.org/10.1073/pnas.1917125117

[6] Bruus, H. (2011). Acoustofluidics 1: Governing equations in microfluidics. Lab on a Chip, 11(22), 3742. https://doi.org/10.1039/c1lc20658c

[7] CAR T Cells: Engineering Patients' Immune Cells to Treat Their Cancers. (2019, July 30). Retrieved February 20, 2021, from https://www.cancer.gov/about-cancer/treatment/research/car-t-cells

[8] Centner, C. S., Murphy, E. M., Priddy, M. C., Moore, J. T., Janis, B. R., Menze, M. A., DeFilippis, A. P., \& Kopechek, J. A. (2020). Ultrasound-induced molecular delivery to erythrocytes using a microfluidic system. Biomicrofluidics, 14(2), 024114. https://doi.org/10.1063/1.5144617

[9] Centner, C. S., Murphy, E. M., Stivers, C. M., Burns, M. S., Priddy, M. C., Janis, B. R., Menze, M. A., \& Kopechek, J. A. (2018, 6-8 Dec. 2018). Development of a high-performance ultrasonic flow system for cell transformation. 2018 IEEE International Symposium on Signal Processing and Information Technology (ISSPIT),

[10] Chen, H., Yu, Z., Bai, S., Lu, H., Xu, D., Chen, C., Liu, D., \& Zhu, Y. (2019). Microfluidic models of physiological or pathological flow shear stress for cell biology, disease modeling and drug development. TrAC Trends in Analytical Chemistry, 117, 186-199.

https://doi.org/https://doi.org/10.1016/j.trac.2019.06.023

[11] DiTommaso, T., Cole, J. M., Cassereau, L., Buggé, J. A., Hanson, J. L. S., Bridgen, D. T., Stokes, B. D., Loughhead, S. M., Beutel, B. A., Gilbert, J. B., Nussbaum, K., Sorrentino, A., Toggweiler, J., Schmidt, T., Gyuelveszi, G., Bernstein, H., \& Sharei, A. (2018). Cell engineering with microfluidic squeezing preserves functionality of primary immune cells in vivo. Proc Natl Acad Sci U S A, 115(46), E10907-e10914. https://doi.org/10.1073/pnas.1809671115 
[12] Hallow, D. M., Seeger, R. A., Kamaev, P. P., Prado, G. R., LaPlaca, M. C., \& Prausnitz, M. R. (2008). Shear-induced intracellular loading of cells with molecules by controlled microfluidics. Biotechnology and bioengineering, 99(4), 846-854. https://doi.org/10.1002/bit.21651

[13] He, Z., Zhang, W., Mao, S., Li, N., Li, H., \& Lin, J. M. (2018). Shear Stress-Enhanced Internalization of Cell Membrane Proteins Indicated by a Hairpin-Type DNA Probe. Anal Chem, 90(9), 55405545. https://doi.org/10.1021/acs.analchem.8b00755

[14] Helfield, B., Chen, X., Watkins, S. C., \& Villanueva, F. S. (2016). Biophysical insight into mechanisms of sonoporation. Proc Natl Acad Sci U S A, 113(36), 9983-9988. https://doi.org/10.1073/pnas.1606915113

[15] Meng, L., Liu, X., Wang, Y., Zhang, W., Zhou, W., Cai, F., Li, F., Wu, J., Xu, L., Niu, L., \& Zheng, H. (2019). Sonoporation of Cells by a Parallel Stable Cavitation Microbubble Array. Advanced Science, 6(17), 1900557. https://doi.org/https://doi.org/10.1002/advs.201900557

[16] Murphy, E. M. (2019). Non-viral transfection efficiencies for the advancement of CAR-T therapy (Publication Number Paper 3248) [Master's Thesis, University of Louisville]. Electronic Theses and Dissertations. https://ir.library.louisville.edu/cgi/viewcontent.cgi?article $=4477 \&$ context=etd

[17] Neelapu, S. S., Locke, F. L., Bartlett, N. L., Lekakis, L. J., Miklos, D. B., Jacobson, C. A., Braunschweig, I., Oluwole, O. O., Siddiqi, T., Lin, Y., Timmerman, J. M., Stiff, P. J., Friedberg, J. W., Flinn, I. W., Goy, A., Hill, B. T., Smith, M. R., Deol, A., Farooq, U., McSweeney, P., Munoz, J., Avivi, I., Castro, J. E., Westin, J. R., Chavez, J. C., Ghobadi, A., Komanduri, K. V., Levy, R., Jacobsen, E. D., Witzig, T. E., Reagan, P., Bot, A., Rossi, J., Navale, L., Jiang, Y., Aycock, J., Elias, M., Chang, D., Wiezorek, J., \& Go, W. Y. (2017). Axicabtagene Ciloleucel CAR T-Cell Therapy in Refractory Large B-Cell Lymphoma. New England Journal of Medicine, 377(26), 2531-2544. https://doi.org/10.1056/nejmoa1707447

[18] Nomikou, N., Tiwari, P., Trehan, T., Gulati, K., \& McHale, A. P. (2012). Studies on neutral, cationic and biotinylated cationic microbubbles in enhancing ultrasound-mediated gene delivery in vitro and in vivo. Acta Biomater, 8(3), 1273-1280. https://doi.org/10.1016/j.actbio.2011.09.010

[19] Perfluorobutane; CAS No. 355-25-9 [Online]; F2 Chemicals: Preston, UK, Feb. 01, 2018. https://www.f2chemicals.com/pdf/sds/Perfluorobutane(355-25-9).pdf (accessed Jan. 2021)

[20] Schneider, T., Hu, Y., Gao, X., Dumas, J., Zorin, D., \& Panozzo, D. (2019). A Large Scale Comparison of Tetrahedral and Hexahedral Elements for Finite Element Analysis. arXiv pre-print server. arXiv:1903.09332v2

[21] Schuster, S. J., Bishop, M. R., Tam, C. S., Waller, E. K., Borchmann, P., McGuirk, J. P., Jäger, U., Jaglowski, S., Andreadis, C., Westin, J. R., Fleury, I., Bachanova, V., Foley, S. R., Ho, P. J., Mielke, S., Magenau, J. M., Holte, H., Pantano, S., Pacaud, L. B., Awasthi, R., Chu, J., Anak, Ö., Salles, G., \& Maziarz, R. T. (2019). Tisagenlecleucel in Adult Relapsed or Refractory Diffuse Large B-Cell Lymphoma. New England Journal of Medicine, 380(1), 45-56. https://doi.org/10.1056/nejmoa1804980

[22] Thermo Fisher Scientific. (n.d.). Electroporation. https://www.thermofisher.com/nl/en/home/references/gibco-cell-culture-basics/transfectionbasics/transfection-methods/electroporation.html

[23] Sharei, A., Cho, N., Mao, S., Jackson, E., Poceviciute, R., Adamo, A., Zoldan, J., Langer, R., \& Jensen, K. F. (2013). Cell squeezing as a robust, microfluidic intracellular delivery platform. $J$ Vis $\operatorname{Exp}(81)$, e50980. https://doi.org/10.3791/50980 
[24] Sharei, A., Zoldan, J., Adamo, A., Sim, W. Y., Cho, N., Jackson, E., Mao, S., Schneider, S., Han, M.J., Lytton-Jean, A., Basto, P. A., Jhunjhunwala, S., Lee, J., Heller, D. A., Kang, J. W., Hartoularos, G. C., Kim, K.-S., Anderson, D. G., Langer, R., \& Jensen, K. F. (2013). A vector-free microfluidic platform for intracellular delivery. Proceedings of the National Academy of Sciences, 110(6), 2082-2087. https://doi.org/10.1073/pnas.1218705110

[25] Shigekawa, K., \& Dower, W. J. (1988). Electroporation of eukaryotes and prokaryotes: a general approach to the introduction of macromolecules into cells. Biotechniques, 6(8), 742-751.

[26] Stone, S. D., \& Hollins, B. C. (2013, 3-5 May 2013). Modeling Shear Stress in Microfluidic Channels for Cellular Applications. 2013 29th Southern Biomedical Engineering Conference,

[27] Tay, A., \& Melosh, N. (2018). Recent Advances in Mammalian Cell Transfection Techniques. postdoc journal, 6(3), 6. https://doi.org/10.14304/SURYA.JPR.V6N3.5

[28] Wang, M., Locke, F. L., Munoz, J., Goy, A., Holmes, H. E., Siddiqi, T., Flinn, I., McSweeney, P. A., Reagan, P. M., Hill, B. T., Jacobson, C. A., Rizzieri, D. A., Heffner, L. T., Jaglowski, S. M., Miklos, D. B., Shaughnessy, P., Unabia, S., Rossi, J. M., Jiang, Y., \& Jain, R. K. (2018). ZUMA2: Phase 2 multicenter study evaluating efficacy of kte-C19 in patients with relapsed/refractory mantle cell lymphoma. Journal of Clinical Oncology, 36(15_suppl), TPS3102-TPS3102. https://doi.org/10.1200/JCO.2018.36.15_suppl.TPS3102

[29] Warboys, C. M., Eric Berson, R., Mann, G. E., Pearson, J. D., \& Weinberg, P. D. (2010). Acute and chronic exposure to shear stress have opposite effects on endothelial permeability to macromolecules. Am J Physiol Heart Circ Physiol, 298(6), H1850-1856. https://doi.org/10.1152/ajpheart.00114.2010

[30] Ward, K., \& Fan, Z. H. (2015). Mixing in microfluidic devices and enhancement methods. $J$ Micromech Microeng, 25(9). https://doi.org/10.1088/0960-1317/25/9/094001

[31] Yu, H., \& Chen, S. (2014). A model to calculate microstreaming-shear stress generated by oscillating microbubbles on the cell membrane in sonoporation. Biomed Mater Eng, 24(1), 861-868. https://doi.org/10.3233/bme-130878 


\section{CURRICULM VITA}

Zachary ("Zach") Long received his Bachelor of Science in Bioengineering from the University of Louisville in 2020. In 2018, he began his first co-op at Cook Medical in Bloomington, Indiana as part of the Vascular Sustaining Team. Throughout his three co-op rotations, Zach was responsible for leading several projects such as process monitoring for the sealing of foil pouches used to package Beacon Tip catheters, implementing leakage test procedures on a line of the Zenith AAA Endovascular Graft products, and various Corrective and Preventative Action ("CAPA") needs related to Flexor Sheaths. His senior capstone project was centered around the creation of test system and method for stickiness of Stryker electrocautery forceps. Following his graduation in May 2020, Zach began completing his Master of Engineering degree in Bioengineering at the University of Louisville under Dr. Jonathan Kopechek and expects to graduate at the beginning of May 2021. Following his passion for medical devices, and more generally, medicine, Zach will begin attending the University of Louisville School of Medicine in July of 2021 to pursue his dream

of becoming a trauma surgeon. Zach has secured a spot as a US Army Health Professions Scholarship Program (“HPSP”) scholar to support his education and goal. 